University of Redlands

\title{
Mobile GIS:
}

The Development of Spatial Analysis Tools in an Integrated Environment for Emergency Responders

A Major Individual Project submitted in partial satisfaction of the requirements for the degree of Master of Science in Geographic Information Systems

\author{
by \\ Nathan Frantz
}

Douglas Flewelling, Ph.D., Director

Bryan Baker, Ph.D., ESRI

December, 2008 


\section{Mobile GIS:}

The Development of Spatial Analysis Tools in an Integrated Environment for Emergency Responders

Copyright $\odot 2008$

by

Nathan P. Frantz 

The report of Nathan P. Frantz is approved.
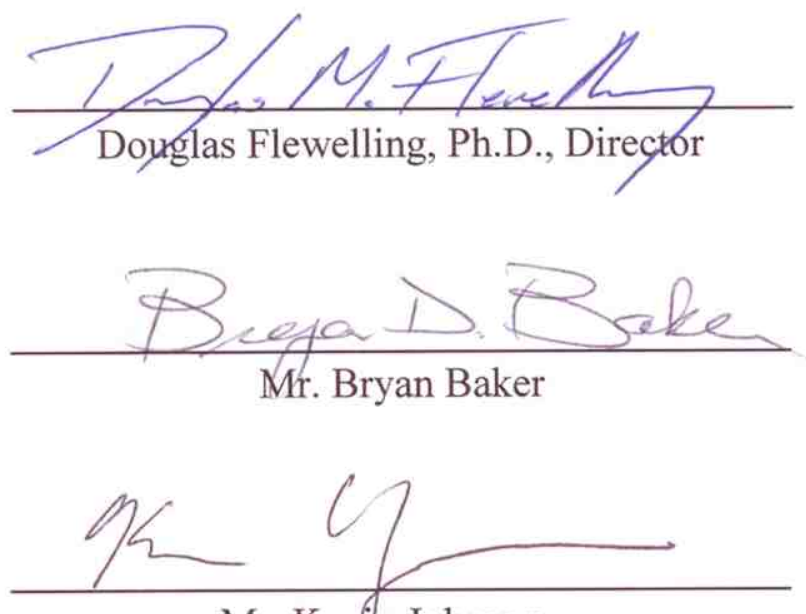

Mr. Kevin Johnson 



\section{ACKNOWLEDGMENTS}

First and foremost my family and fiancé Anissa Jamison deserve tremendous credit for providing the support needed in this intense year.

ESRI was also instrumental to the success of this project during the development phases. In particular the mobile development team assisted in providing the necessary resources and base development of the project application. In particular Myles Sutherland and Frederick Aubry provided the contacts and resources to team members who were able to define the project scope to complete the defined requirements. Mike Shaw played an integral role in this with assistance in developing a custom web-based service to complete the geoprocessing tasks. Later application development was also supported by Mark Deaton, of the Applications Prototype Lab, who helped define and develop geoprocessing service requirements, enabling the final pieces of the project to be completed. Finally, Anthony Fragale, who has developed a majority of the existing PREACT mobile software, assisted in the development of core geometry-based functions within the $\mathrm{C} \#$ programming environment.

Finally, the Army Corps of Engineers, Topographic Engineering Center (TEC), and the project client provided the knowledge and assistance to guide the project as requirements changed. Mr. Kevin Johnson, the project client, is very knowledgeable in the project domain and assisted in defining the requirements for successful completion. Mr. Aaron Bernard also assisted with this process, as well as editing the project documentation for its technical competence within PREACT. Mr. Bernard is a technical lead within the PREACT project and provided a great deal of insight into the development and subsequent inclusion of these tools within PREACT.

The committee for this project also deserves a great deal of appreciation for editing and guiding the final project documentation. Dr. Bryan Baker of ESRI provided excellent feedback in the subject matter and has a great deal of experience with ArcGIS Server implementation. I also would like to thank Dr. Douglas Flewelling for his assistance as the chair of the committee as well as his support as an advisor throughout the project cycle. 




\begin{abstract}
Bringing GIS to the field has many limitations to the end user due to currently available mobile computing and connectivity shortcomings. Of major concern is the lack of spatial analysis and querying abilities currently available in commercial off-the-shelf (COTS) software for mobile applications. Current mobile GIS offers mapping and visualization capabilities, while providing no analytical means to discover the data spatially. Adding spatially-based analysis tools will enable emergency responders to more accurately record the state of a particular area and better understand their surroundings while collecting important field assessments. This allows for more timely and accurate assessments by emergency planners to better allocate resources in the field. This paper outlines the development of both connected or server run, and disconnected analysis tools for emergency responders in a mobile framework.

The proposed tools were developed with ESRI's ArcGIS Server Mobile Software Development Kit utilizing server technology. They leverage the mobile application based data and internet-based services to perform these tasks. This project is focused on the development, and subsequent inclusion into, an enterprise emergency response system: PREACT.
\end{abstract}





\section{Table of Contents}

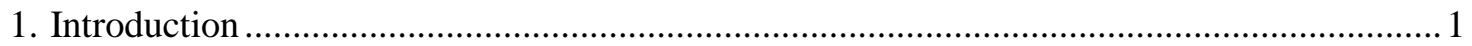

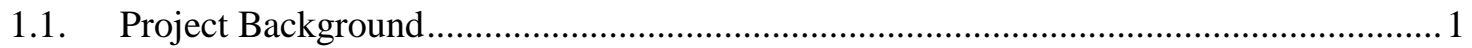

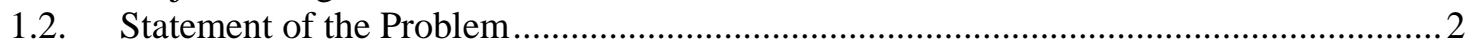

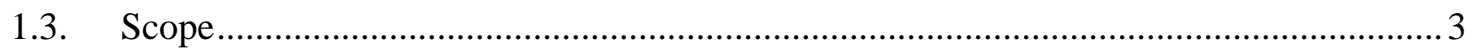

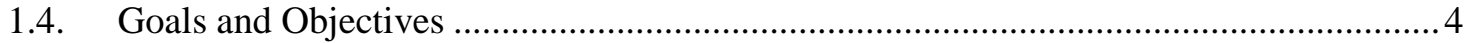

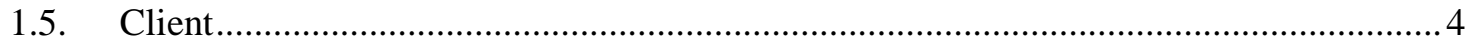

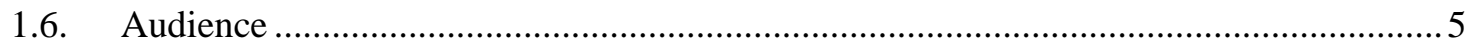

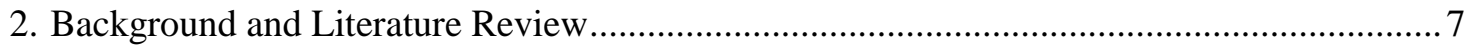

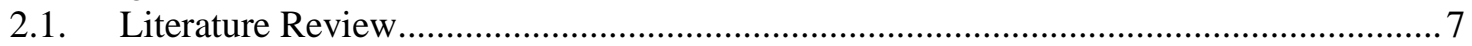

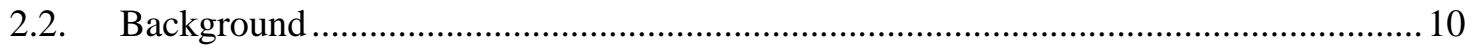

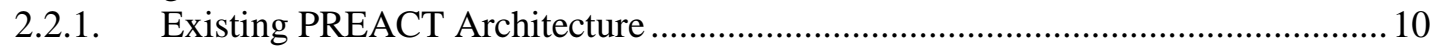

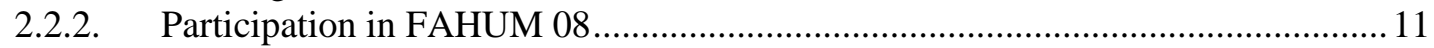

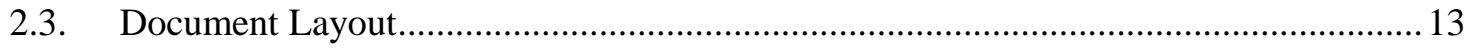

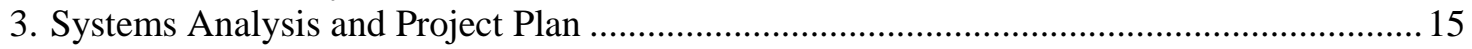

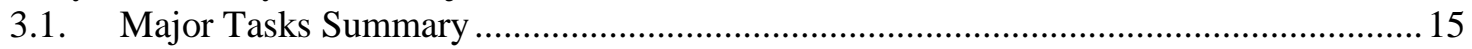

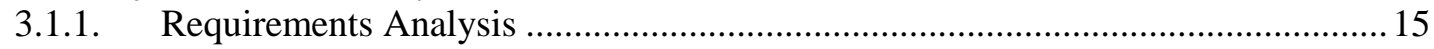

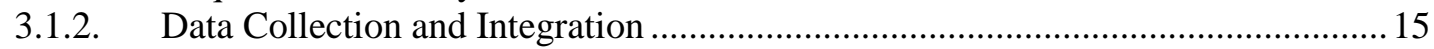

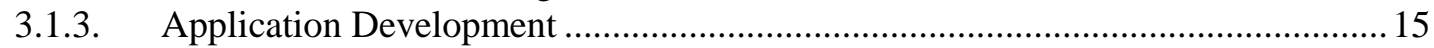

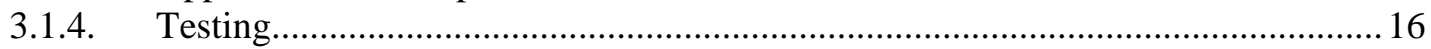

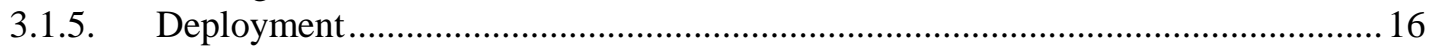

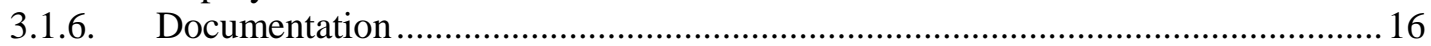

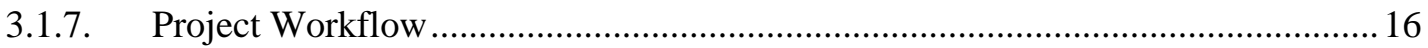

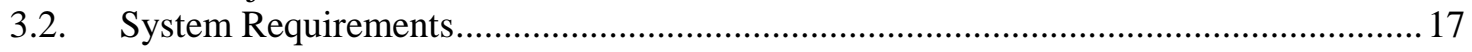

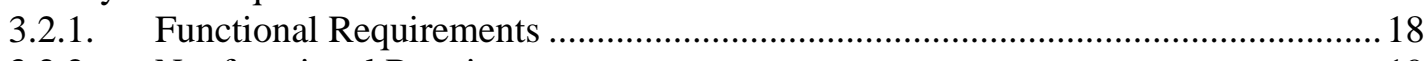

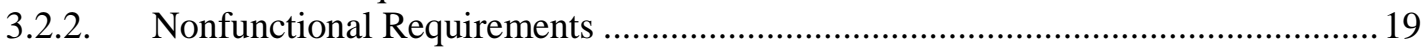

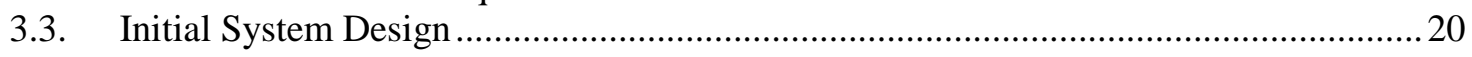

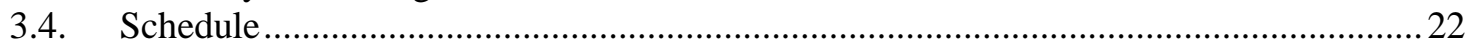

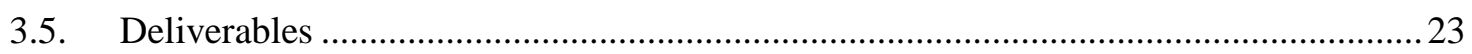

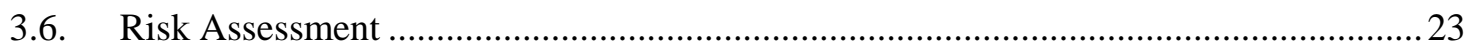

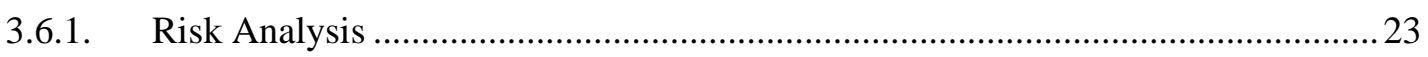

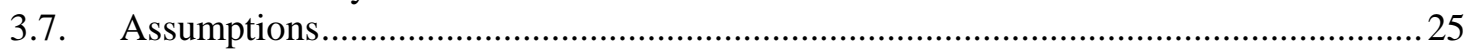

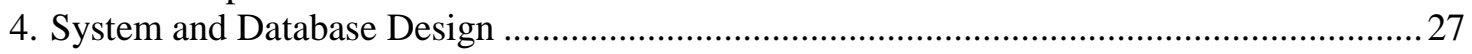

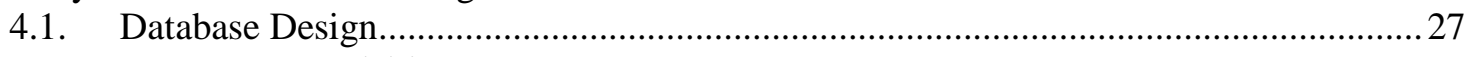

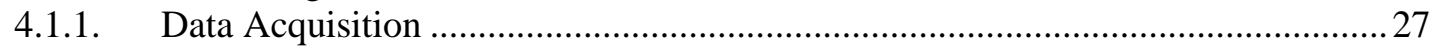

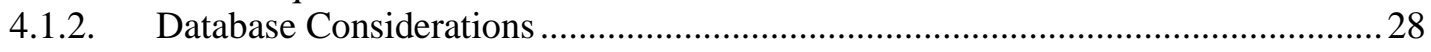

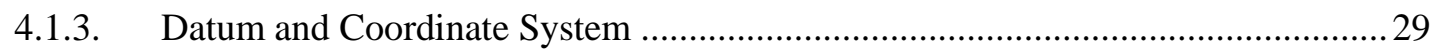

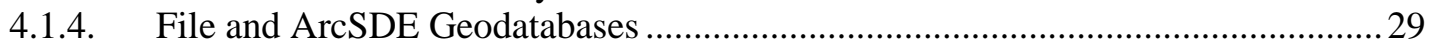

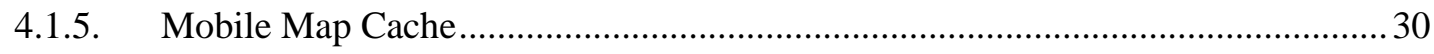

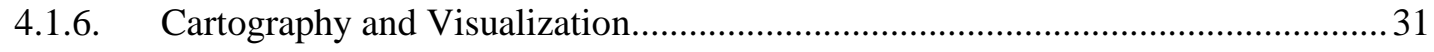

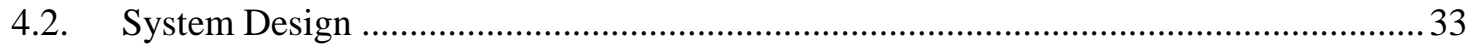

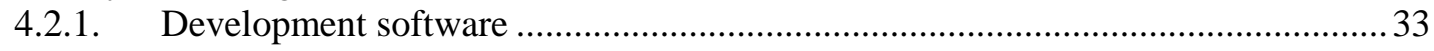

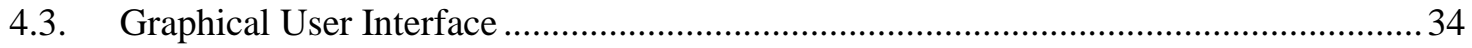

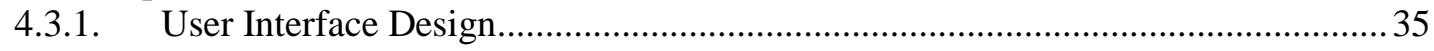

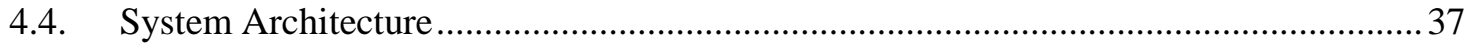

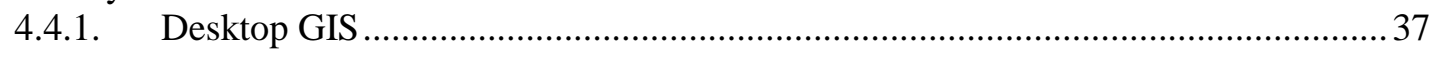

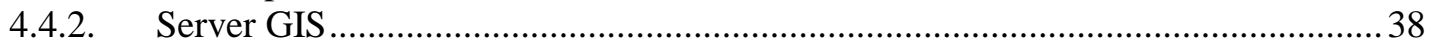




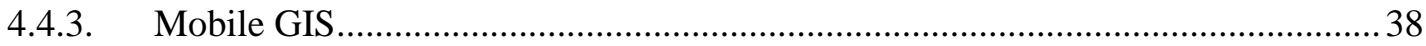

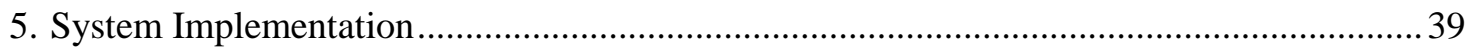

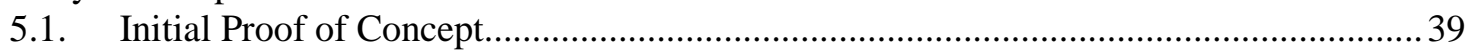

5.2. Modified Sample Mobile Geoprocessing Application............................................... 39

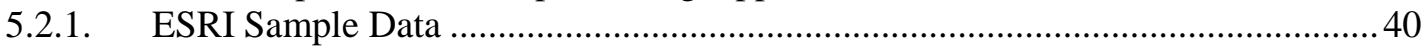

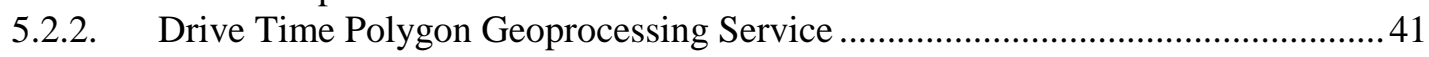

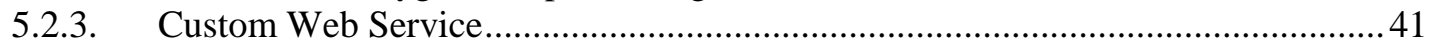

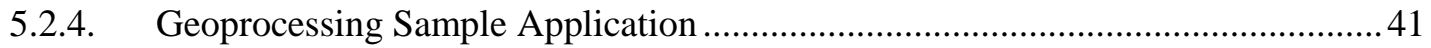

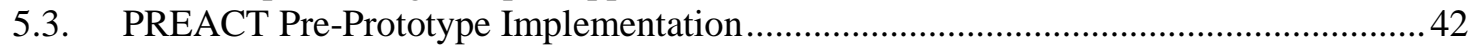

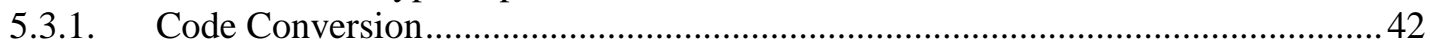

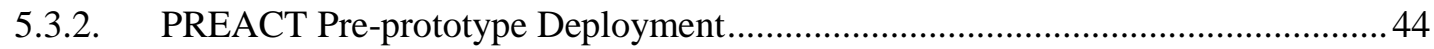

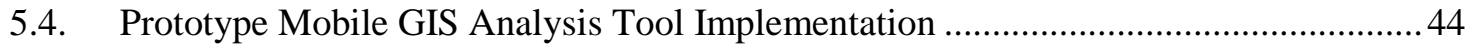

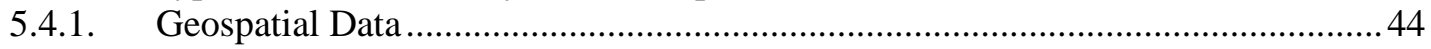

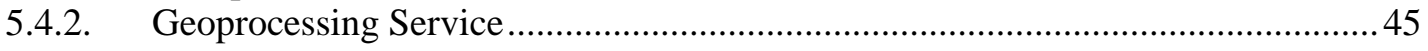

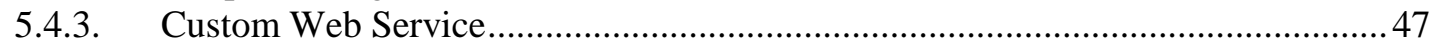

5.4.4. Mobile Analysis Tool Software Development and Deployment ............................49

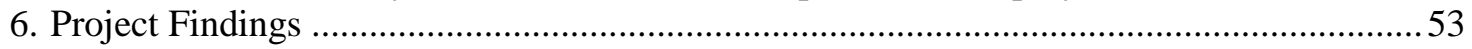

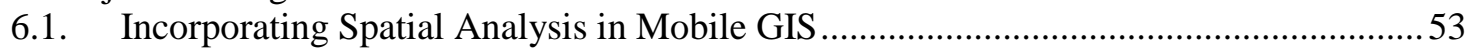

6.1.1. Limitations of Geoprocessing Services in ArcGIS Server Mobile .........................53

6.1.2. Triggering Geoprocessing Tasks from a Mobile Client......................................54

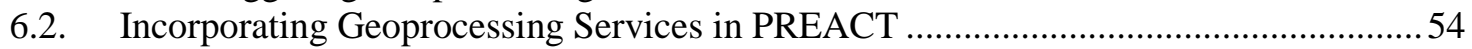

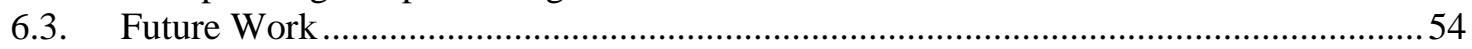

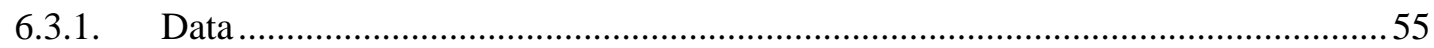

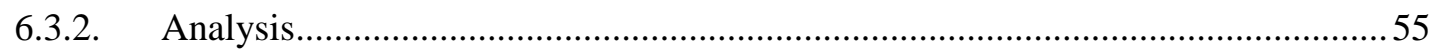

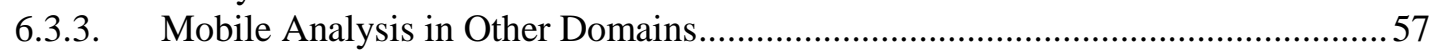

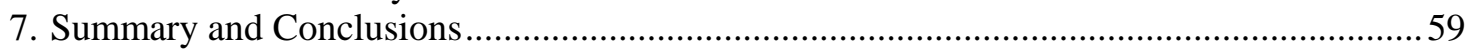

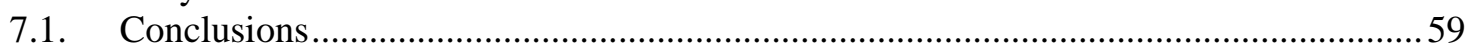

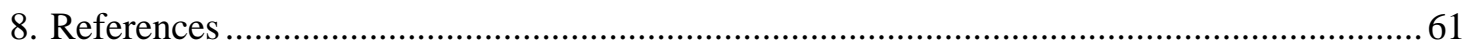





\section{List of Figures}

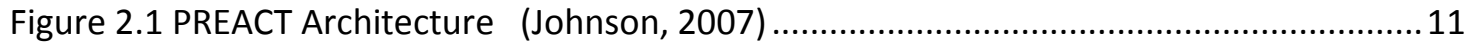

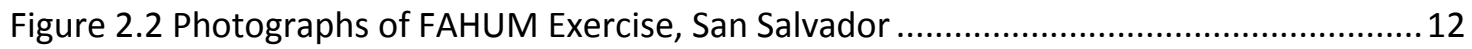

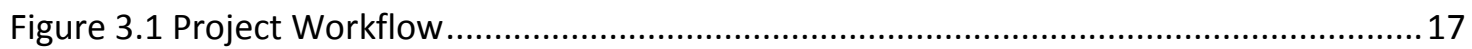

Figure 3.2 Mobile System Data Storage and Analysis ........................................................ 21

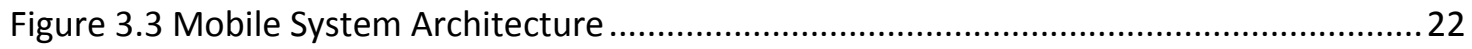

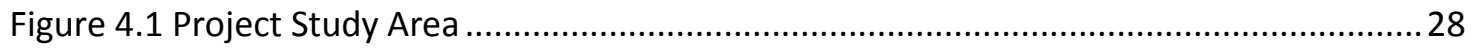

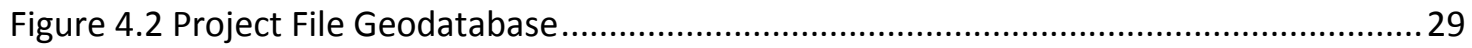

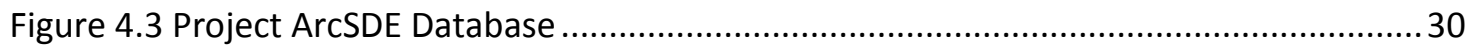

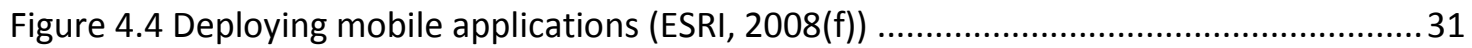

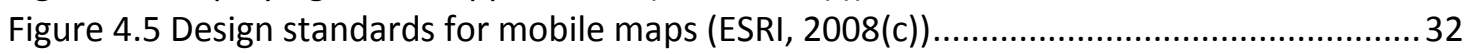

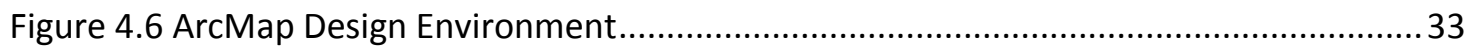

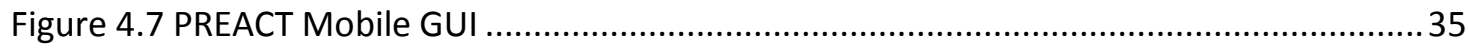

Figure 4.8 Existing and newly design PREACT mobile interface ......................................... 36

Figure 4.9 Common Mobile Mapping Interfaces Showing User Interface Designs (Google, 2008) (ESRI,

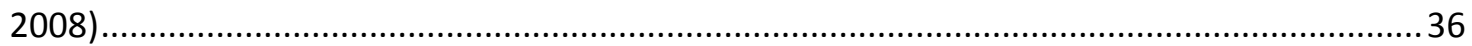

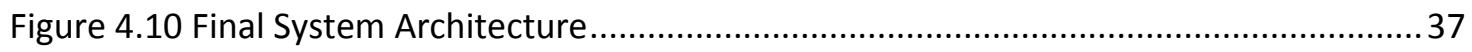

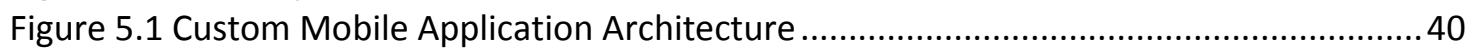

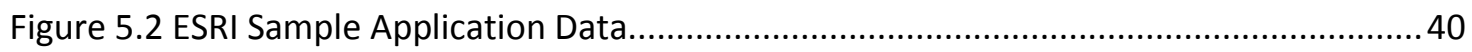

Figure 5.3 Modified ESRI Sample Application Interface and Model Output .............................42

Figure 5.4 Sample Geoprocessing task runnning in PREACT Mobile.......................................44

Figure 5.5 Sample LANDSCAN data for San Bernardino County ...........................................45

Figure 5.6 Population Summary Model (ESRI 2008) ........................................................... 46

Figure 5.7 Population Summary geoprocessing service REST resource (ESRI, 2008(a)) ..........47

Figure 5.8 Project Interface and Analysis Tool Control ...................................................... 49

Figure 5.9 User Feedback for Polygon Creation ................................................................. 50

Figure 5.10 Final Project Output for Population Summary Analysis ......................................51 




\section{List of Tables}

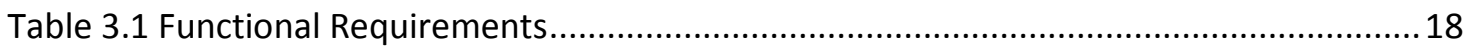

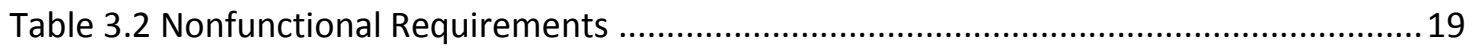

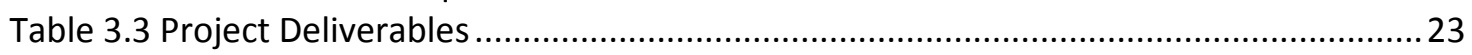

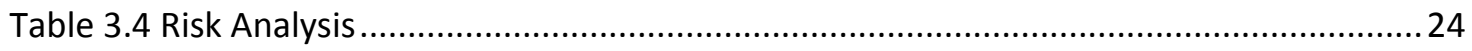






\section{List of Acronyms}

AOR

C\#

DOD

ESRI

EOC

FAHUM

GIS

GUI

HTTP

MXD

PREACT

SDE

SDK

SOAP

SQL

SSF

SSTR

TEC

TOC

US SOUTHCOM

VB.NET
Area of Responsibility

C-Sharp

Department of Defense

Environmental Systems Research Institute

Emergency Operations Center

Fuerzas Aliadas Humanitarias

Geographic Information System

Graphical User Interface

Hypertext Transfer Protocol

ArcMap Document

Preplanned Emergency Response and Action

Spatial Data Engine

Software Development Kit

Simple Object Access Protocol

Structured Query Language

Simple Spatial Data Format

Stability, Security, Transition, and Reconstruction

Topographic Engineering Center

Table of Contents

United States Southern Command

Visual Basic .NET 



\section{Introduction}

The ability to collect and analyze emergency response assessment data in a GIS context will allow for more timely decisions and aid in the event of a major disaster. Incorporating field specialists, technology, and a GIS can aid in this process through developing mobile applications for field responders. These applications can in turn provide more accurate actionable intelligence to the decision makers throughout the course of the emergency response phase. This actionable intelligence allows users to better identify areas that are in need of specific services and products.

The main objective of this project is to provide a technical solution to increase the use of spatial information in a disaster area through real actionable intelligence and analytical capabilities. To achieve these stated objectives, an enterprise system implementing a centrally managed and accessible system for mobile application development was completed. This mobile application allows for updates to the enterprise system, for use by all key facilitators in the emergency management process. This spatially based mobile application allows for further development of spatial analysis tools available to the field collection teams. These analysis tools will run on both the central server through connected geoprocessing services, as well as on the mobile device, through the disconnected software. This is a key requirement, as the austere conditions in the focus area will likely lack sufficient communications networks to allow for a permanent connection to the central server.

\subsection{Project Background}

Policy guidance in Department of Defense Directive 3000.05 allows for military support of Stability, Security, Transition, and Reconstruction (SSTR) efforts (Department of Defense, 2005). This initiative has allowed the US Army Corps of Engineers Topographic Engineering Center (TEC) to work with Central American partner nations to develop a new GIS-based system for emergency response and disaster management. TEC, in coordination with US Southern Command (SOUTHCOM), began development of the Preplanned Response and Emergency Action (PREACT) project on October 1, 2006. PREACT directly supports the DOD directive by increasing the regional stability in the US SOUTHCOM area of responsibility (AOR) through a coordinated planning and response capability for emergency managers. The PREACT project “... will enable accurate assessments, situational awareness, dynamic planning, and synchronized response to international disasters." (Watts, 2007)

The disaster response capacity currently in place in many of these partner nations relies on a pen and paper-based system of data collection. This information is then verbally disseminated back to the Emergency Operations Center (EOC) by cellular phone and entered into various databases for cataloging and analysis. This system results in the 
ineffective timeliness of updates to situational awareness, as well as directing future field assessment team distribution. It also renders decision support ineffective when trying to

view and analyze data from these multiple disparate databases. As a result, the PREACT project has implemented an enterprise GIS system with mobile collection capabilities. This mobile application allows disaster responders to collect and visually display geographically related data, as well as assessment data. This mobile application will be the focus for this project.

All stages of emergency response and disaster management use GIS, including: response, preparedness, mitigation, and recovery (Raheja, Ojha, \& Mallik). Creating a system that encompasses these four aspects of the disaster management cycle is key in addressing all deficiencies in the process. One deficiency of particular importance is the response phase's field assessment capability, which is crucial in identifying areas of need after a disaster. This functionality not only addresses the field assessment itself, but also how that data is synthesized by the disaster responders for better spatial awareness in the response area.

Because of the inherent spatial nature of this problem, as well as the current state of GIS technology, this problem effectively solves the problem by employing a geospatial solution. Therefore, the focus of this research and resulting project will center on the use of mobile software development to address the objectives of PREACT, SOUTHCOM, and partner nations regarding enhanced field analytical capabilities. These software tools will work in coordination with the existing data collection and mapping mobile application available in PREACT.

\subsection{Statement of the Problem}

In the event of a disaster, field assessment teams are sent into a response area to collect data on the current conditions on the ground. Through PREACT, TEC has developed the ability to view this spatial data and collect assessment data digitally within the geospatial framework on a mobile device.

This development represents advancements in the current state of technology used for assessment teams and has built a framework that allow for new processes to be developed, further strengthening the disaster responder's data collection and spatial awareness abilities. PREACT addresses this issue with mobile data collection and mapping software. However, the PREACT software does not address the ability to provide real time spatial analysis based on the team's current location during the data collection process. "A GIS is most often associated with a map. A map, however, is only one way you can work with geographic data in a GIS, and only one product generated by a GIS. A GIS can provide a great deal more problem-solving capabilities than using a simple mapping program..." (ESRI, 2008). Emergency responders are left without spatial analytical capabilities, a significant component in GIS. The ability to query and analyze the GIS database and collected field data will provide the final step in the mobile GIS component of PREACT. 


\subsection{Scope}

The intended project involved developing prototype spatial analysis tools using ArcGIS Mobile and ArcGIS Server. The development was done in the Visual Basic .NET programming environment and incorporated into the existing PREACT Mobile system that the client provided. The system utilizes web services in a network environment with a mobile software interface provided by the client. Additionally, the project develops the mobile capabilities for improved spatial awareness in two ArcGIS Mobile modes: connected and disconnected.

This document outlines the use of mobile GIS to provide spatial analytical tools within the following areas:

1. Time Frame: 10-month period to develop and document all tools

2. Location: San Bernardino, California

3. Analyses:

a. Trigger geoprocessing tools residing on ArcGIS Server

b. Run simple selection-based tools with a mobile device in a disconnected environment

The tools will utilize built-in GPS technology on the mobile device as appropriate and will have the ability to generate output from the analysis tools. The output will be either in table format or displayed as a selection set on the mobile map.

The client provided data for their study area allowing for a data collection base for the project. San Bernardino County, California was chosen as the area of interest (AOI) for the project because of the data detail, the quality of spatial data available, and the need for local GPS readings during the development phase of the project. Checking the supplied data for completeness to identify any needed collection areas or missing data was also necessary. A file geodatabase and Spatial Data Engine (SDE) database organized this data for use with ArcGIS Server.

The final deliverable consists of the application in an executable form for use on Windows mobile-based devices. All source code used to develop the project will be included with the spatial database(s) developed from the collected data. Once delivered to the client, it will be the responsibility of the Topographic Engineering Center to decide how and when the application will be incorporated into PREACT or other projects for use in disaster management. All deliverables are in digital format and available on DVD.

The initial project scope was overestimated in several areas, resulting in several tasks not being completed. This was due mostly to the given project time frame and unforeseen issues in the development phase. This limited the scope to focus only on the connected geoprocessing tools with future work in the design and implementation of the disconnected tools. 


\subsection{Goals and Objectives}

The main objective of this project was to provide a technical solution to increase the use of spatial information in a disaster through spatially-based analytical capabilities. The mobile GIS application provides an easier and more accurate data collection framework. This is compared to the current paper and pencil disaster assessment workflow by partner nations in the SOUTHCOM AOR of. The overall success of the project will be based on the ability to provide analytical capabilities to disaster responders on a mobile platform. Mobile GIS performs this as part of an enterprise GIS, which replicates and disseminates collected field assessment data to multiple end users (for example other disaster responders). It is unlikely that responders in a disaster area will have access to data networks (cellular or Wi-Fi); thus, the solution must be able to analyze the cached data on the mobile device while disconnected from a network. The client's previous work on the PREACT project was incorporated and the focus of this project was to further develop spatial analysis tools for mobile users.

As will be discussed in future chapters, incorporating typically web-based geoprocessing services are leveraged by a mobile application running ArcGIS Mobile software. These geoprocessing tasks reside on the central server, allowing for connected mobile devices to trigger geoprocessing tasks and return the model output to the field. This was a significant accomplishment in the project development, allowing for access to tools and data not stored locally on the mobile device.

\subsection{Client}

The client for this project is Mr. Kevin Johnson of the Topographic Engineering Center. Mr. Johnson is an Associate Technical Director, as well as the Deputy Project Manager and Technical Lead on PREACT, with an extensive background in enterprise GIS as a manager of several disaster response and preparedness projects for federal organizations.

Mr. Johnson has supported this project by:

- Providing technical support in applying GIS to disaster management primarily through the mobile development phase of the project.

- Aiding in the acquisition of data and specifying the need for new data from Central American governments and agencies.

- Assisting in defining and narrowing the scope and purpose of the project so it not only uses an innovative domain of GIS technology, but also addresses a legitimate need of partner nations for improved disaster response systems.

- Providing contacts for partner nations, who will in turn provide additional requirements and feedback for the project

- Identifying other domains for which this developed technology may be applicable to, such as DODs Humanitarian Assistance projects. 


\subsection{Audience}

This paper is intended for a professional audience with a background in emergency response and management, GIS, or both. The end user will benefit from this project in the areas of mobile spatial analysis and emergency response capabilities for disaster responders.

Emergency Responders should take away the vision of how leveraging mobile GIS can assist them during the response phase of a disaster. GIS professionals and developers will have a better understanding of the process required to implement a spatial analytical tool for mobile users through ArcGIS products. 


\section{Background and Literature Review}

\subsection{Literature Review}

When considering mobile GIS development, several key disciplines were researched to assess and design a mobile application that fully encompasses the need of its designated application in disaster management. Currently, field assessments are collected using paper and pencil and then phoned or radioed back to an EOC. This system exposed a large deficiency in communications and the ability to view real time information regarding the response area. Emergency management data is inherently spatial (Cova, 1999) and is not adequately addressed in current GIS processes. The ability to collect data in a spatial format and subsequently disseminate that data in a standards-based architecture has increased the ability to understand fully the current state of an emergency. "GIS provides a mechanism to centralize and visually display critical information during an emergency" (Johnson, May 2000).

GIS is not a new tool being implemented in emergency management, but has already aided in disaster management with a host of new technologies, most notably the ability to create, analyze, and disseminate geospatial information in an enterprise environment. "The value of GIS in emergency management arises directly from the benefits of integrating a technology designed to support spatial decision making into a field with a strong need to address numerous critical spatial decisions" (Cova, 1999). GIS played a significant role in the disaster response at the World Trade Center on September 11, 2001. "Remote sensing and GIS were used to develop preliminary damage assessments - at gross scales and by individual building and/or infrastructure" (Cutter, 2003). The ability to collect these preliminary damage assessments is a prime function of the PREACT mobile software. This will enable the collection of spatial information, as well as increase the spatial awareness of each disaster responder who uses the software. FEMA has also introduced the use of GIS in emergency management with its All-Hazard Situation Assessment Program (ASAP), which was designed to support the short-term assessment collection process (Linz and Bryant, 1994, Cova, 1999).

Understanding the full capability of applying mobile GIS to data collection is a key resource for identifying its role in disaster management. Tsou (2004) outlines the importance of mobile GIS solutions in location-based services, and in field-based GIS. Field-based GIS refers to the collection and manipulation of spatial data, while locationbased systems are used for routing and navigation (Tsou, 2004). Both systems play a role in defining an appropriate system to meet the requirements of responders in disaster management. In the event of a disaster, the ability to adequately navigate through the response area, as well as collect and disseminate data based on observations in that area provides a value-added service to everyone involved in the process. "For the geoscientists, the use of mobile computers can facilitate data collection and also provide a means for discovering data relationships while still in the field" (Vivioni \& Camilli, 2003). 
The implication of these mobile services is the change from static mapping and processes to dynamic services that are location-based. "...many of the items we want to locate are far from static - they move - and there is significant value in knowing when, how and how quickly they move, and the direction and route taken."(Winnie S. M. Tang, 2003) This particularly plays a role in emergency management and how disaster responders receive information in the field. The ability to dynamically update and query location-based information allows for faster response times and relevant updates to disaster teams. "This demands the ability to capture location information on moving objects and events, and to transmit this, in real-time, to recipients who may themselves be moving." (Winnie S. M. Tang, 2003) This process encompasses the need for service-based operations that allow access to data and spatial analytical tools typically found in GIS.

Cutter (2003) identifies several shortcomings in GI Science regarding its application to disaster management, including: spatial data acquisition and integration, distributed computing, dynamic representation of physical and human processes, cognition of geographic information, and interoperability. A mobile application has the ability to address each of these challenges, particularly the ability to collect spatial data and integrate that data dynamically back to the support system in near real time. Mobile software would also significantly increase the "cognition of geographic information" (Cutter, 2003) by the field user through mobile mapping. "The vast majority of disaster responders (such as police, fire, emergency medical personnel) are not familiar with GIS, nor are they likely to use these systems in the immediate response or rescue phase." (Cutter, 2003) This is a major concern in developing a mobile system for collecting assessment data. Disaster responders will need systems that are rugged, easy to carry, and, most importantly, easy to use. The goal of mobile data collection in early phases of a disaster is limited to the data necessary to better enable decision makers, without placing any extra burden on disaster responders, whose ability to adapt to new technology will hinge on an easy-to-learn user interface which better enables their mission (Cutter, 2003).

Many of these shortcomings are the result of limitations in the hardware and software available on mobile-based devices. "In spite of advantages of Mobile GIS, it is difficult to support very large-volume GIS spatial data in mobile environments because mobile computing environments have several limitations such as - low processing power, low memory, small screen size, limited communication environments, and so on."(Jong-Woo Kim, 2004) Kim and others examined three types of mobile GIS applications: applicationbased, server-based, and HTML-based. Significant performance gains can be seen in application-based mobile GIS through the reduction of spatial data. This was accomplished by restructuring the data in a Simple Spatial Data Format (SSF). Along with the size reduction of the spatial data, server-based HTTP/SOAP protocols were also implemented which allowed for processing to be done on the server. The combination of the two provided an efficient means of handling large spatial datasets and rendering on mobile devices. 
The ability to effectively communicate disaster-related data in a spatial format will provide emergency managers and workers alike an enhanced view of ground conditions during the recovery and response phase of a disaster. Mobile technology has already proven its worth in field data collection, therefore showing its potential in addressing this communication deficit to dynamically disseminate and integrate spatial data. "There are significant trends that govern the future of response and recovery in this country, including, but not limited to the development of new technologies (remote sensing, geographic information systems (GISs)), modeling, decision support systems, etc.;" (Hecker, Irwin, Cottrell, \& Bruzewicz, 2000) This new trend seems to have a strong footing in mobile application development which is based in a GIS to effectively collect data.

Several existing and developing technologies that incorporate GIS into mobile platforms were also examined. ESRI has long been involved in this industry with its ArcPad application, which allows for basic mapping and data collection in the field. "ArcPad provides field-based personnel with the ability to capture, analyze, and display geographic information, without the use of costly and outdated paper map books." (ESRI, 2008(h)) Although this product allowed for mapping functionality in the field, it did not offer a capability to remain connected to an enterprise system where dynamic updates could be made from the field. This requires a user to physically dock their mobile device in order to post data, through ArcGIS desktop to the central database. Due to the operating conditions in post-disaster, this technology has serious limitations that ESRI addressed with its ArcGIS Mobile software suite. "ArcGIS Mobile is a mobile GIS platform that enables organizations to deliver geographic information system (GIS) capabilities and data from centralized servers, providing access to GIS services over wireless networks to a range of mobile devices." (ESRI, 2008(i)) This technology is based on ArcGIS Server, allowing for dynamic data updates while in the field. This new technology was a major advancement in the ability to collect and update emergency-related data that is immediately posted to a central database. ArcGIS Mobile, however, is limited to Windows Mobile 5 or 6-based devices. Another company, TDC Group, has also developed mobile software now available for use on BlackBerry devices, which are also based on ArcGIS Server. "Freeance Mobile ${ }^{\mathrm{TM}}$ quickly brings location to enterprise data and maps on BlackBerry® Smartphone's. Enterprises can now use rich, up-to-date data from their enterprise GIS and back to office databases in mobile applications on BlackBerry Smartphone's that are useful, cost-effective and easy-to-use.” (TDC Group, 2008)

The implication of these new service-oriented mobile applications is the ability to leverage data and services that are not included on the device itself. This allows for more robust applications and unlimited amounts of data available to the field user without compromising the limited hardware configurations of mobile devices. The ability to leverage server-based GIS while in the field greatly reduces the hardware and software demands of mobile devices.

Of particular importance to this new development in server computing is the development of communication networks available for mobile devices. "Prior to the widespread emergence of mobile communication services the work on mobile computer systems has tended to focus upon the development of small devices and the forms of interaction that 
they afford." (Tom Rodden, 2000) These new communication channels have allowed server-based processing and data to be accessed from mobile devices, most notably smart phones. This new technology has advanced the mobile computing abilities to not reside solely on the device, and to expand the capabilities and storage shortfalls of the past. Rodden discusses the importance of location in mobile devices on the context of this new infrastructure. "The maturing of technology to allow the emergence of multiuser distributed applications that exploit mobile applications means that we can no longer focus the issues of interaction on the nature of the device."(Tom Rodden, 2000) In other words, mobile applications can now be designed to interact with other entities, with the context of location as a major consideration. Location is a major concern when incorporating GIS and spatial analysis into mobile computing.

It is also important to note that because of the new technology being used in this project, there are limited resources detailing how such systems have been implemented in the past. This created new challenges for this project which needed to be addressed in a scientific manner to assure that the proposed solution was suitable in addressing the stated problem. With these tools comes a need for a redesigned framework and interface available to mobile users.

\subsection{Background}

Since this project is developed with the existing PREACT project, it is important to define the current PREACT project and system architecture. In particular, the PREACT mobile application served as a basis for this research and the subsequent project upon which all tools were developed and will be deployed, too, if accepted by the client.

\subsubsection{Existing PREACT Architecture}

The PREACT mobile application was created in response to the lack of a sufficient field collection method to update key players during the response phase of a disaster. This new technology would not only standardize the collection process, but also update the current situation in near real time. The application was initially developed with capabilities in ArcGIS Server 9.2 which contained a new mobile application SDK for creating customizable Windows and Windows Mobile-based applications.

This new software allowed the PREACT developers to customize and develop a field collection software package that was tightly coupled into a larger GIS enterprise. This enterprise consists of a central Database Management System (DBMS), server technologies, proprietary and open source desktop applications, web-based clients, and web services. Figure 2.1 is an illustration of the initial PREACT architecture. The focus of this architecture and subsequent system was to disseminate information to various key players, unconstrained by a specific technology to eliminate the "stovepipes" that are common in many emergency response systems. 


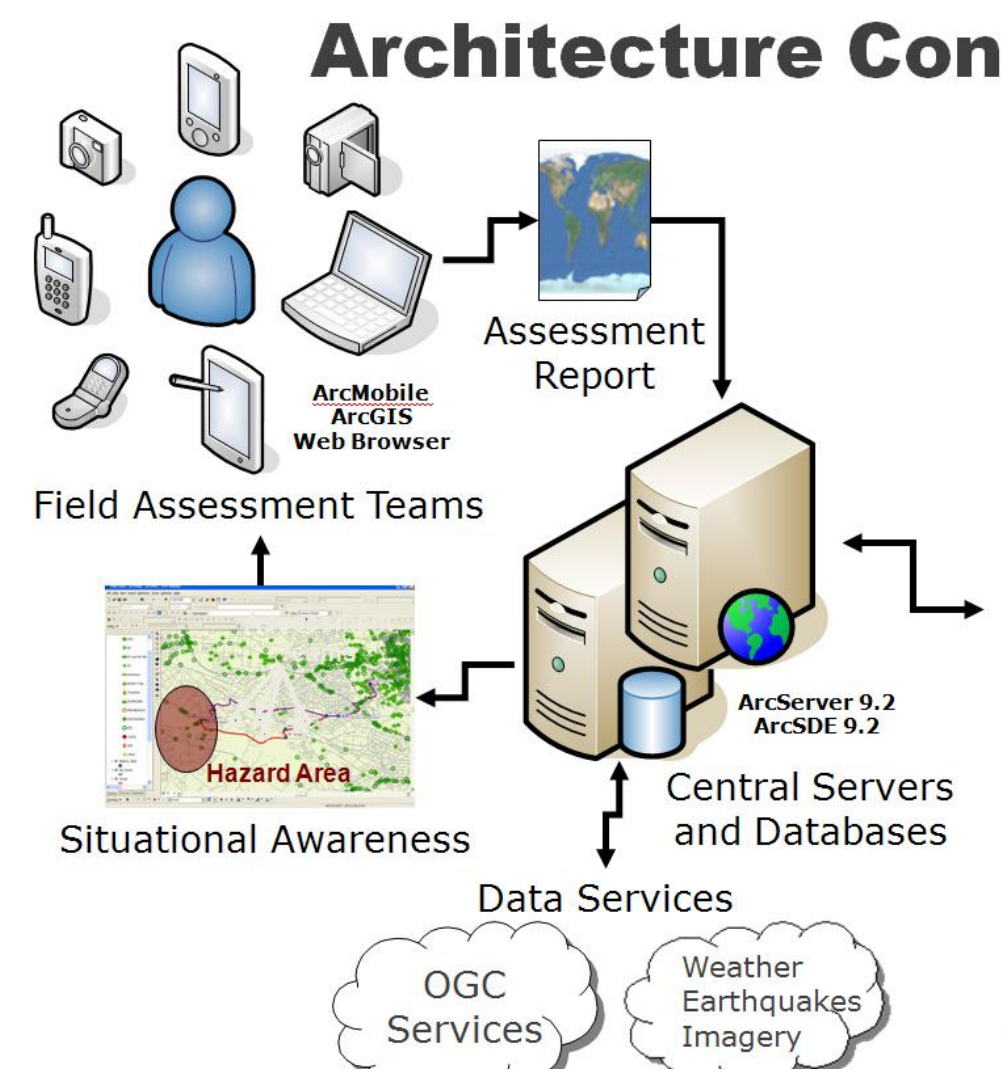

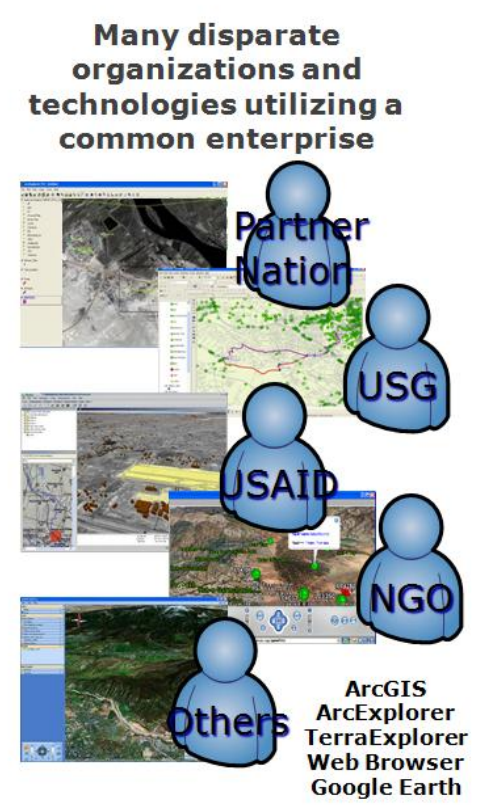

Pre and Post Event Activities at Operation

Figure 2.1 PREACT Architecture (Johnson, 2007)

Through this initial system, the mobile capabilities were added to enable the field collection capabilities used by partner nations. This first mobile application was designed for situational visualization and as a platform to collect geographic information with the capability to synchronize back to the central database server.

\subsubsection{Participation in FAHUM 08}

Every year an exercise the Fuerzas Aliadas Humanitarias (FAHUM) is conducted in Central American countries to demonstrate and work on emergency response-related technologies and procedures. "FAHUM is a tabletop exercise that allows personnel to sharpen and practice their skills in order to be better prepared to respond to future challenges, while bringing lasting benefits to the people of the participating nations and strengthening our enduring partnership."(US Southern Command, 2007) This exercise and conference allows for all participants involved in the emergency management for these countries to showcase and more accurately define software applications for emergency response. In 2008 this conference was held in San Salvador, El Salvador, as a two-week event with a full week of an operational emergency response exercise. This exercise was attended by most of the Central American countries, as well as many United States organizations and government 
entities, including the US Army. Figure 2.2 shows photographs taken during the exercise in a small village just outside San Salvador where assessment collections took place. The PREACT software was tested by military personnel during the exercise to determine its data collection and situational awareness capabilities. The field user (right image) aided in the discovery of features that would further the collection process and discussed some of the analytical capabilities he thought would assist him in responding to disasters.
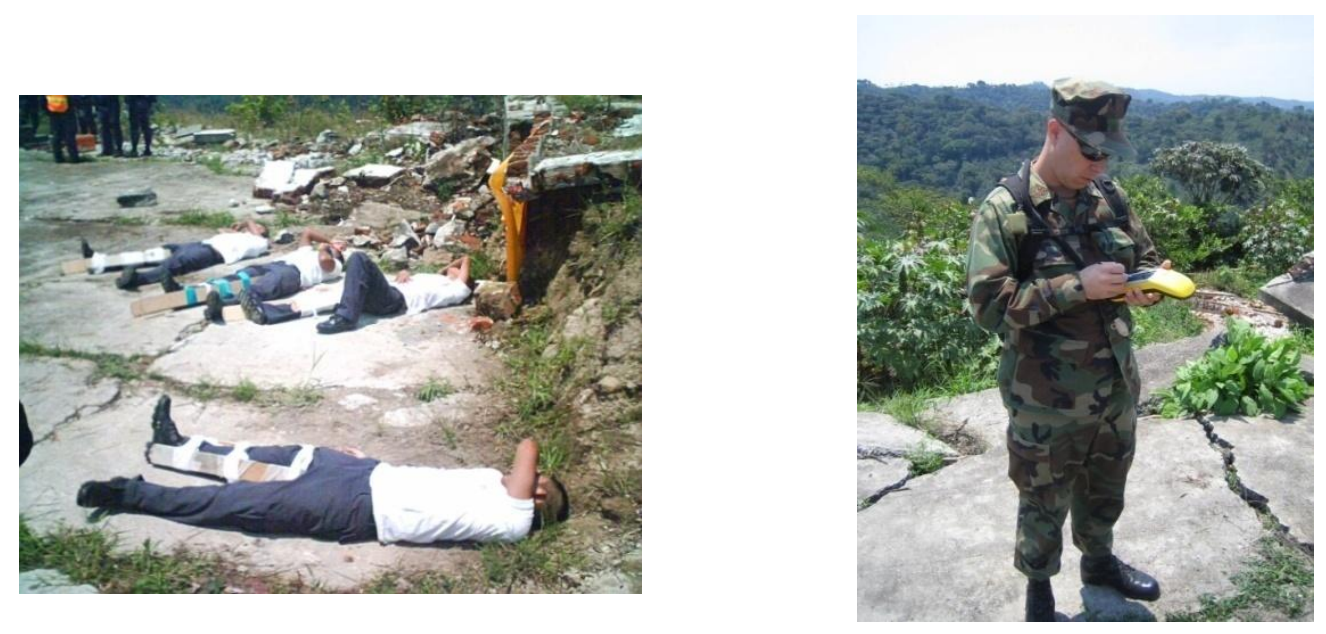

Figure 2.2 Photographs of FAHUM Exercise, San Salvador

The feedback from this needs assessment (Appendix A), facilitated the development of the requirements that served as the framework for the emergency management functionality and tools that would be useful to assist in assessing a disaster. Below is an excerpt from that list of recommendations. Of particular importance was the need to quickly retrieve general population counts to support decisions regarding potential victims. This would in turn be reported in the assessments sent back to the EOC and compiled to aid in allocating resources and personnel.

- Needed on the Assessment form:

○ Total population counts (while the general population may not be in need of immediate services, an estimate of total people in the village/area would be very beneficial)

- Hazard Classification

○ More detailed information obtained from the USAID Field Operations Guide

The ability to see the working environment and take part in an emergency response exercise provided invaluable insight about how disaster responders do their jobs. This experience provided a firsthand experience of the conditions and possible hindrances for disaster responders after an event. Turning these experiences and lessons into valuable tools was the next logical step in better aiding the collection process. 


\subsection{Document Layout}

The remainder of this document outlines the processes and procedures performed to complete the described project. Chapter Three serves as a description of how the project will be implemented through a series of system analysis and design procedures. The following chapter outlines the system design and database design used to develop the mobile tools. The fifth chapter describes the implementation and methodology undertaken to produces the mobile analysis tools. The final two chapters serve as a summary and future consideration for the project, and include the relevant work which was not completed in this project due to time constraints. 


\section{Systems Analysis and Project Plan}

Prior to development, project plan and system analyses were performed to outline the major tasks necessary to complete the project requirements. Six major tasks were identified and carried out in cycles to address the analytical capabilities needed for PREACT. This plan, as well as the accompanying requirements and documentation, outline these major tasks.

\subsection{Major Tasks Summary}

The following project life cycle was utilized by the author to complete the project with input from the project client. This lifecycle incorporated the following stated tasks that were employed in a spiral development phase. This approach allowed for multiple tasks to be ongoing at the same time as new objectives and requirements were discovered.

\subsubsection{Requirements Analysis}

A requirements analysis was undertaken to outline the functional requirements, as well as any possible risks and mitigations for successful completion of the project. Specifying the project requirements provided the criteria for project completion. This task outlines the client's needs within the constraints of the PREACT projects objectives. This includes specifying the software and hardware to be used in this project, which are already being used by the client.

1. Identify client needs

2. Identify gaps in the collection process

3. State the objectives and goals of the project

4. Specify software and hardware to be used in the project

\subsubsection{Data Collection and Integration}

All data from the PREACT project was supplied by the client, some of which was originally obtained from Central American governments. Although these data were not used in the project, they provided the basis for developing representative data sets. The database schema was based on past emergency management database designs, including the working PREACT schema. It was decided that the data supplied by the client would not be appropriate for this project due to testing issues and disseminating the data outside of the PREACT project.

\subsubsection{Application Development}

This project focused on developing custom tools not available in COTS. A majority of the project work occurred during this cycle with the development of custom spatial analytical tools for use on Windows-based mobile devices. The ESRI technologies do not offer outof-the-box tools so extensive programming was required during development, and required research into any current work ESRI. This research was ongoing throughout the project development to keep abreast of new technologies offered by ESRI that would insure 
successful integration of the proposed tools. The geoprocessing tool integration and simple analysis tools were also designed to resemble the look and feel of existing ArcGIS desktop tools, which provide similar functionality to desktop users.

Developing the application started with the configuration of all existing PREACT software and server architecture to replicate the enterprise system the client has in place. Once this was completed new map and geoprocessing services were generated from the collected project data. These services were used in the development phase for the mobile application development for access to data and models residing on ArcGIS Server. The project data was also used to generate a map cache for storing each feature class in the ArcMap map document (MXD) used for the project. Finally, geoprocessing tools were developed using these components, allowing users to access spatial tools residing on the project server.

\subsubsection{Testing}

To insure the tools worked as planned, the testing cycle was ongoing during the application development. This cycle, a large part of the project development, insured that the project was on track and meeting the client's needs. Prototype tools were developed to test their functionality in aiding the field assessment teams. These prototype applications were deployed to mobile devices and tested for functionality. The final testing phase entailed a look at how the tools work according to the project requirements laid out prior to development.

\subsubsection{Deployment}

Once the tools were considered satisfactory by the client, they were included in a final application build that will run on Windows mobile-based devices. This entailed a final executable .cab file deployed to any Windows-based device and the final map cache. The deployment of these tools will allow for the client to test and examine their functionality. As a final step the client can then make the decision on what to include in the PREACT application, which will be used in response to disasters.

\subsubsection{Documentation}

The last major task for the project was a final document, including all processes and conclusions from the project development. As a significant portion of the project requirements, this task was a major portion of the project with significant contributions focused on its completion. The final documentation was worked on throughout the entire project and included a final project write-up, final presentation, and all deliverables handed off to the client.

\subsubsection{Project Workflow}

The major tasks outlined above are reflected in the project workflow below. The key requirements and potential decision points are outlined. The project planning and requirements were conducted to outline what the project would do and to insure that the client's requests were met. The development and testing phases were a major 
consideration in the project development and were monitored by the author and client to comply with the project plan. As each stage of the project was completed documentation was compiled for the clients review. The final documentation consisted of these concurrent update reports, as well as the results and conclusions once the project was considered to be completed. (Figure 3.1)

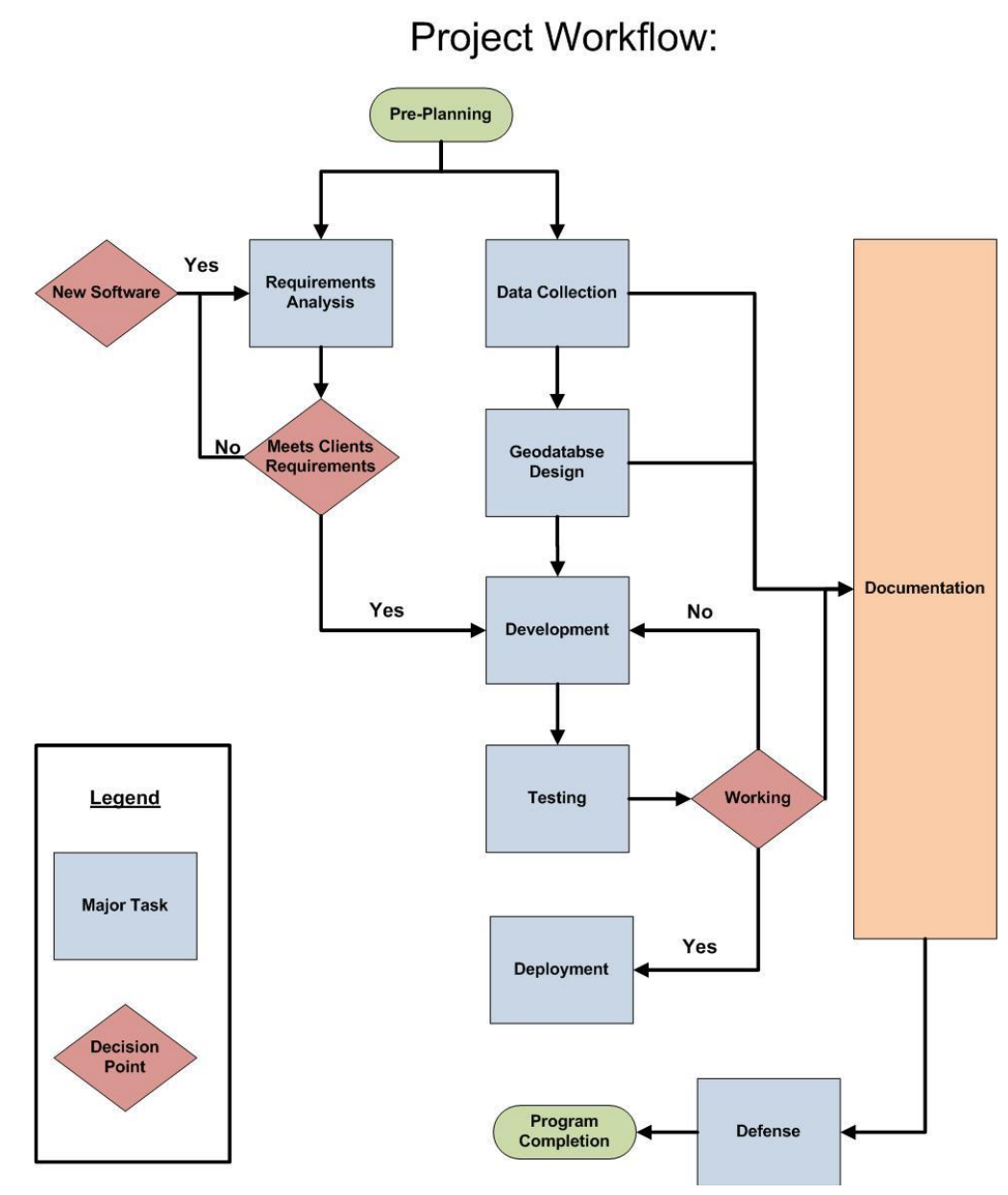

Figure 3.1 Project Workflow

\subsection{System Requirements}

As a basis for completion in the allotted time frame, a set of system requirements were developed outlining the tasks included in project development. These requirements were broken down into two key areas: functional and non-functional. The functional requirements outline what the system will do, while the non-functional requirements serve as a basis for how the system behaves. These requirements were adhered to, as well as used to quantify the results of the prototype project. Functional requirements were broken down into four key development areas which closely followed the project life cycle including: the overall system, database, data access, and application development. The 
non-functional requirements are grouped as behavioral constraints which have been outlined for use in the application development. (See Table 3.1)

The key requirement of this project was to provide web services for mobile applications which leverage spatial analytical capabilities. This was mandatory within the overall system, along with incorporation of devices with built-in GPS which could be used for location based analytics. It was also desirable to have analysis capabilities in connected and disconnected environments because of the limited network connectivity in disaster conditions. As addressed later, only server-based tools were implemented due to development issues.

The database and data access requirements of the project were driven by the existing PREACT project. All data acquired for the study area represented the available data for the PREACT development team. While the project did not look at advancing the current state of the PREACT database design, recommendations were made to improve their current system. This also included a look at how to best implement cartographic rules to display this data on a mobile device.

\subsubsection{Functional Requirements}

Table 3.1 Functional Requirements

\begin{tabular}{|c|c|c|}
\hline 1 & Overall System & \\
\hline 1.1 & Leverage services managed through a central server & Mandatory \\
\hline 1.2 & $\begin{array}{l}\text { Provide analytical tools to mobile clients through the use of server-based } \\
\text { enterprise system }\end{array}$ & Mandatory \\
\hline 1.3 & Support portable handheld devices with integrated GPS & Mandatory \\
\hline 1.1 & $\begin{array}{l}\text { Provide geospatial analytical tools in a connected and disconnected } \\
\text { environment }\end{array}$ & Desirable \\
\hline 1.5 & Provide the ability to visually display spatial analysis results and overlays & Desirable \\
\hline 1.6 & Perform all development work in Visual Basic .NET using ArcGIS Server & Desirable \\
\hline 2 & Database & \\
\hline 2.1 & $\begin{array}{l}\text { Provide a data model and storage capability for spatial analysis specific data } \\
\text { locally in bin format. The data will need to be stored in a spatial format with a } \\
\text { shape field and unique object ID }\end{array}$ & Mandatory \\
\hline 2.2 & $\begin{array}{l}\text { Leverage existing databases used in the PREACT project. This will require } \\
\text { the use of spatial databases in a file geodatabase format }\end{array}$ & Mandatory \\
\hline 2.3 & Provide the retrieving, updating, and storage of analysis data & Desirable \\
\hline 3 & Data Access & \\
\hline
\end{tabular}




\begin{tabular}{|c|c|c|}
\hline 3.1 & Create mobile services for data synchronization and replication & Mandatory \\
\hline 3.2 & $\begin{array}{l}\text { Provide the capability to store, index, search, and manage outputted data from } \\
\text { the analysis tools in bin format readable by the mobile device for spatial } \\
\text { visualization }\end{array}$ & Desirable \\
\hline 3.3 & $\begin{array}{l}\text { Develop cartographically appropriate mobile-based maps for use in mobile } \\
\text { services which will be visually simple in design with minimal amounts of data } \\
\text { to allow for limited space requirements }(<200 \mathrm{MB})\end{array}$ & Desirable \\
\hline 4 & Application & \\
\hline 4.1 & Support mobile devices operating on the Windows Mobile 5.0 platform & Mandatory \\
\hline 4.2 & $\begin{array}{l}\text { Connected tools will leverage geoprocessing services running on central } \\
\text { server }\end{array}$ & Mandatory \\
\hline 4.3 & $\begin{array}{l}\text { Provide the capability to transmit and receive data via existing standard data } \\
\text { communication transfer mechanisms and protocols on the mobile device (e.g. } \\
\text { Wi-Fi, Bluetooth, cellular, etc...) }\end{array}$ & Mandatory \\
\hline 4.4 & Incorporate asynchronous server calls to run geoprocessing models & Desirable \\
\hline 4.5 & $\begin{array}{l}\text { Develop a deployable executable application which can be installed on any } \\
\text { Windows Mobile } 5.0 \text { device }\end{array}$ & Desirable \\
\hline 4.8 & Provide the ability to generate reports for outputted analytical data & Desirable \\
\hline 4.9 & Ability to buffer features & Desirable \\
\hline
\end{tabular}

\subsubsection{Nonfunctional Requirements}

The desirable nonfunctional requirements outline the behavioral qualities of the system design for end users. These requirements were meant to drive the project interface and interaction with the system. Since the primary goal of the project was to develop a prototype application, these requirements were not as important in development, leading to many of them being deemed optional to the final product. (See Table 3.2)

Table 3.2 Nonfunctional Requirements

\begin{tabular}{|r|l|l|}
\hline 1.0 & Leverage existing COTS software to develop the analysis tools and interface & Desirable \\
\hline 2.0 & Provide end users an intuitive graphical user interface to access these tools & Desirable \\
\hline 3.0 & $\begin{array}{l}\text { The system will be reliable and work under the rigors of field use and harsh } \\
\text { environments of field exercises in the event of a disaster }\end{array}$ & Desirable \\
\hline 4.0 & $\begin{array}{l}\text { The analytical tools can be tailored to the users desired output by using open- } \\
\text { ended input and output layers }\end{array}$ & Optional \\
\hline
\end{tabular}




\begin{tabular}{|r|l|c|}
\cline { 2 - 3 } 5.0 & The system will run in both English and Spanish & Optional \\
\hline 6.0 & Ability to upload and download analytical data in a low bandwidth architecture & Desirable \\
\hline 7.0 & $\begin{array}{l}\text { Analytical tools must not be so advanced that they compromise the mobile } \\
\text { hardware and software }\end{array}$ & Desirable \\
\hline 8.0 & $\begin{array}{l}\text { Security-based features for sensitive data with general login and user } \\
\text { verification }\end{array}$ & Optional \\
\hline
\end{tabular}

\subsection{Initial System Design}

The system was designed with ArcGIS Server 9.3 from the Environmental System Research Institute (ESRI). ArcGIS Server offered an enterprise solution upon which the central geodatabase and necessary services interacts with mobile clients, running the PREACT software with built-in analysis functionality. The design process incorporated the following functions based on this architecture:

1. Map and data authoring

2. Server database, application, and services created/implemented

3. Mobile map cache and application developed

4. Server-based geoprocessing services developed

5. Disconnected analysis tools developed

These analysis tools run on the mobile device, as well as through the geoprocessing services residing on the server. These geoprocessing services will provide for the mobile and web clients with an interface to spatial models authored in ArcGIS Desktop. The models and model data reside locally on the server and only the model output is returned to the end user.

The secondary focus of this project was to incorporate the disconnected analysis tools, which run solely on the mobile device. These analysis tools utilize the map layers which will first be authored in Desktop and then served out to the mobile devices via the map services from ArcGIS Server. All data on the mobile device was deployed in the map cache generated on the Server as .bin files, and include any analysis results run in the disconnected environment (Figure 3.2). These analyses and their corresponding locally stored output data will update the enterprise geodatabase upon synchronizing the map cache. 


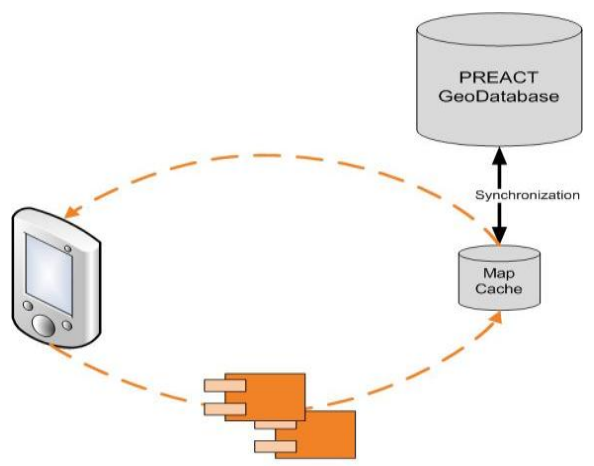

Figure 3.2 Mobile System Data Storage and Analysis

The system architecture (Figure 3.3) outlines this process and the general flow of data and processes. ArcGIS Server will be the backbone of the system, providing the services and database management for the mobile device. The mobile application will serve as the key component of this project, making use of the services and application to provide analytical capabilities to the end user. 


\section{Mobile Analysis System Architecture}

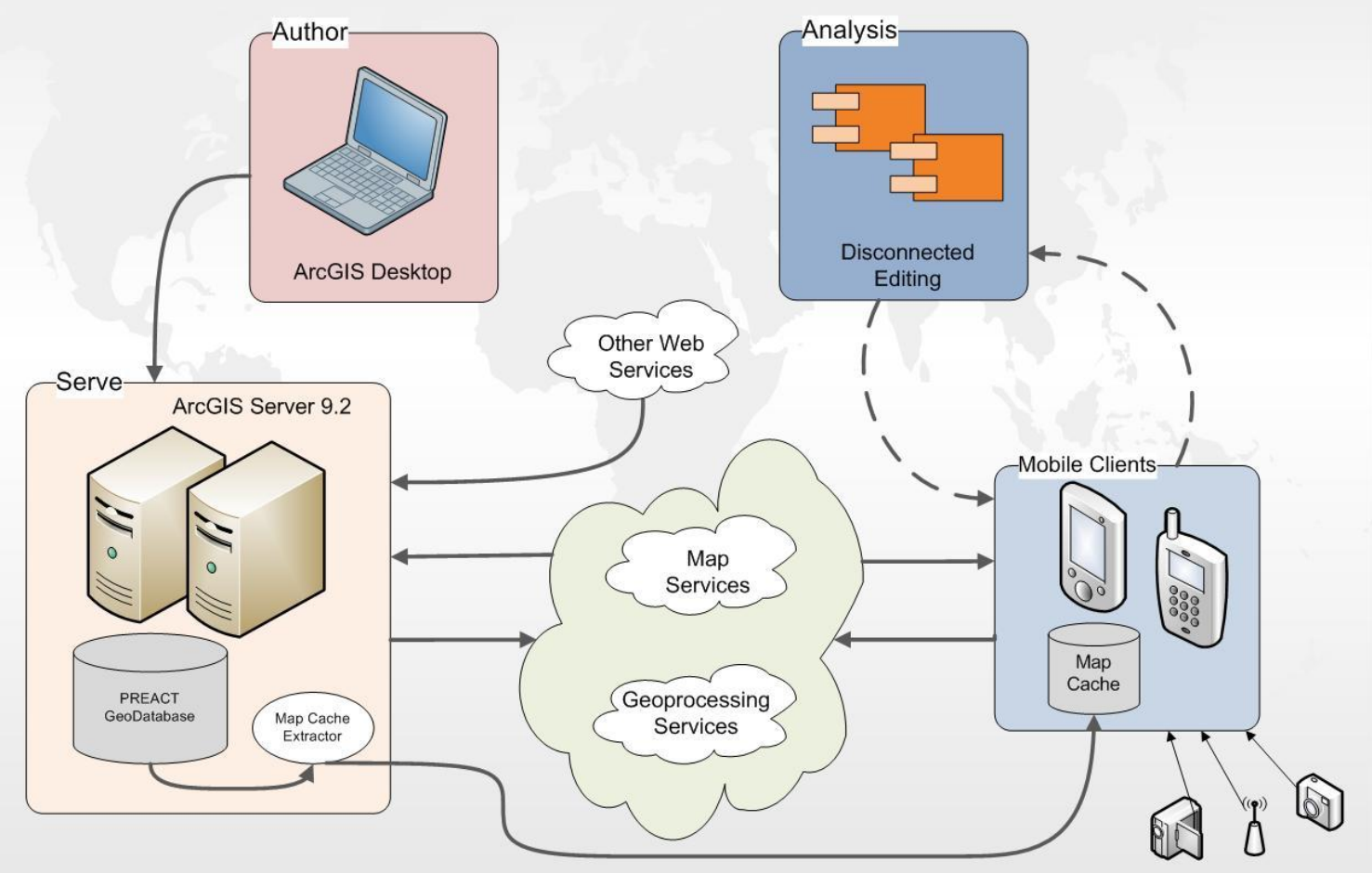

Figure 3.3 Mobile System Architecture

\subsection{Schedule}

With a one year time frame to complete the stated project, a schedule was developed to guide the phases of development and project work. This schedule gives a general outline of when tasks were completed and the approximate amount of time spent on each cycle (see Appendix B).

Major efforts were put into the development and testing phases of this project. This task consumed a majority of the time allotted and subsequently limited other phases of the project. This was acceptable as the tools and processes developed are a prototype and more testing and development is necessary before they reach a deployable phase.

Also of importance was the project documentation which was a significant requirement for the project. This included the final project write-up and a defense presentation to discuss the development and processes undertaken to achieve the stated tasks. These requirements, along with the developed tools, were required to be completed no later than December 4, 2008 for the client's review. All work was handed off to the client after it was considered satisfactory. 


\subsection{Deliverables}

The following table outlines the final products needed to satisfy the client's requirements, as well as intermediate milestone deliverables. The intermediate deliverables are intended to aid in the timeline of the project and inform the client of current progress. This includes a bi-weekly status report, project proposal, and completed deliverables.

Table 3.3 Project Deliverables

PROJECT Deliverable Schedule

\begin{tabular}{lclc}
\hline \multicolumn{1}{c}{ Deliverable } & Maturity & Due Date & Recipient \\
\hline Biweekly status report & Final & Biweekly & Client \\
Project Proposal & Draft & $21-$ Feb-08 & Client \\
Completed Geodatabase & Draft & $25-$ Apr-08 & Client \\
Prototype Code & Draft & 1-Aug-08 & Client \\
Final Presentation & Final & 25-Nov-08 & All \\
Final Deliverables & Final & 28-Nov-08 & All \\
\hline
\end{tabular}

\subsection{Risk Assessment}

The following risk assessment addressed the possible issues and known risks which may have contributed to or the prevention of successful completion of the proposed project. The purpose of this document was to outline the possible risks and attempts to find solutions to accomplish the assigned project in the time given.

\subsubsection{Risk Analysis}

Before development could begin it was essential to outline some of the key risks which could possibly change the project schedule. It was necessary to plan for these risks and have mitigation strategies in place if they did play a role in the project development.

In particular, since the ArcGIS Mobile software is so new, ESRI continues to release updates which add to the functionality available out-of-the-box. In the early stages of project planning ArcGIS Server 9.3 was being released and it was unclear as to how the mobile framework would change. Once ArcGIS Server 9.3 was released it was discovered that the entire mobile SDK was changed, prompting the client to convert all of the existing code to 
the 9.3 environment. This risk had a high exposure rate, requiring the initial proposal to be changed to only implement the server based geoprocessing tools.

The other identified risks outline the potential issues which typically plague research and prototype application when dealing with new software which has been vetted by users.

The following table outlines these risks and the potential exposure before development began, with their associated mitigation tactics. The final project completion saw most of these risks come to fruition through many development issues, including ESRI software and test hardware used in the project

Table 3.4 Risk Analysis

\begin{tabular}{|c|c|c|c|c|}
\hline Risk & Severity & Probability & Mitigation & Exposure \\
\hline $\begin{array}{l}\text { New } \\
\text { Technology } \\
\text { (ArcGIS 9.3) }\end{array}$ & 4 & 5 & $\begin{array}{l}\text { Keep abreast of possible new } \\
\text { technologies when defining project } \\
\text { scope } \\
\text { - Limit the ability of incorporating } \\
\text { new technology in the project }\end{array}$ & 20 \\
\hline $\begin{array}{l}\text { Inexperience } \\
\text { with } \\
\text { Programming }\end{array}$ & 4 & 4 & $\begin{array}{ll}\text { - } & \text { Schedule ESRI training to better } \\
\text { understand the language } \\
\text { - } \\
\text { Purchase VB programming } \\
\text { material }\end{array}$ & 16 \\
\hline Scope Creep & 3 & 4 & 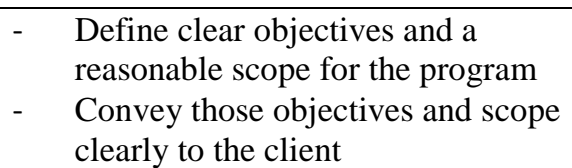 & 12 \\
\hline $\begin{array}{l}\text { Hardware Issues } \\
\text { (Server, mobile) }\end{array}$ & 2 & 3 & $\begin{array}{l}\text { Add hardware profiles to the } \\
\text { project scope }\end{array}$ & 6 \\
\hline Software Issues & 2 & 3 & $\begin{array}{l}\text { - Limit the project to only the } \\
\text { software currently needed to } \\
\text { complete the project } \\
\text { Only use software the client has } \\
\text { worked with in the past }\end{array}$ & 6 \\
\hline
\end{tabular}

$*$ Scale $=1-5,5$ being the most severe

Of significant importance throughout the development and planning stages was the high likelihood of new software from ESRI. Project development with this new software could have potentially undermined the mobile analysis tools with out-of-the-box solutions to the proposed analysis tools. This would have drastically changed the course of the research and subsequent final project. There were also significant concerns regarding insufficient programming experience to complete all the tasks. The rest of the listed risks were not as 
likely or as severe to the potential project development setbacks but were worth mentioning.

\subsection{Assumptions}

To properly mitigate possible roadblocks during the project completion, assumptions were made to identify these potential setbacks. Understanding these assumptions and communicating them to all parties involved in the project assisted in the proper mitigation of these assumptions before they became a problem. Below is a list of these identified assumptions:

- The client will provide adequate assistance to aid in the programming and development phase of the project.

- Replicating the PREACT architecture and server design locally is possible

- Assistance will be available from ESRI personnel regarding the structure and possible inclusion of spatial analysis within the Mobile SDK framework

- Mobile SDK framework will support the proposed analysis tools for the project

- Mobile hardware and software will be able to handle the new processing tasks from the inclusion of the alerting and spatial analysis tools

- Client will return all deliverables in a reasonable time frame

Based on these assumptions, several factors contributed to a delay in certain project development areas. Of particular importance was the ability to trigger geoprocessing tasks on the server from a mobile device. This was initially thought to be a simpler task due to a demonstration from ESRI, which allowed for access to a geoprocessing service from ArcGIS Mobile. This demo was a one-off application with custom web services and code to make the geoprocessing tasks work as described. This issue led to a much longer development cycle for the geoprocessing tasks to be developed. This was partially mitigated by several meetings and discussions with the Mobile Development Team at ESRI, which allowed for the custom web services and code to be developed from the demo application to the PREACT application. 


\section{System and Database Design}

A significant portion of the project work was devoted to the system and database design. This applied to both the new system design for the analysis tools, and re-creating the existing PREACT system design and architecture for testing and development purposes.

\subsection{Database Design}

PREACT's current database design provided a basis for organizing this project's data. This included a file geodatabase and an SDE database for base data and analysis layers. ArcCatalog was used to create, manage, and clean these geodatabases within the desktop environment. This represented most of the PREACT enterprise system re-creation.

\subsubsection{Data Acquisition}

Data was collected for the San Bernardino County, California, area based on the PREACT database schema. This area was chosen because of the requirement to include locationbased services where GPS testing would be critical. This data was also readily available and would not need to be cleared by the client for use within this project or the countries who originally supplied the data. Figure 4.1 is a detailed map of this study area and selected data.

Although more data were available for the area, it was important to limit the data collection and resolution for use as surrogate data for the existing PREACT database. These considerations were examined for the analytical capabilities for use in the intended PREACT area of interest. For example, street addressing would not be possible in many Central American countries and therefore the project did not include these data even though it was readily available.

Most of the base data collected for the project is available from ESRI. This includes base layers available within their Data and Maps product. Many of these layers are available both nationally and worldwide, which would afford the PREACT team access to these layers for incorporating the project tools. 


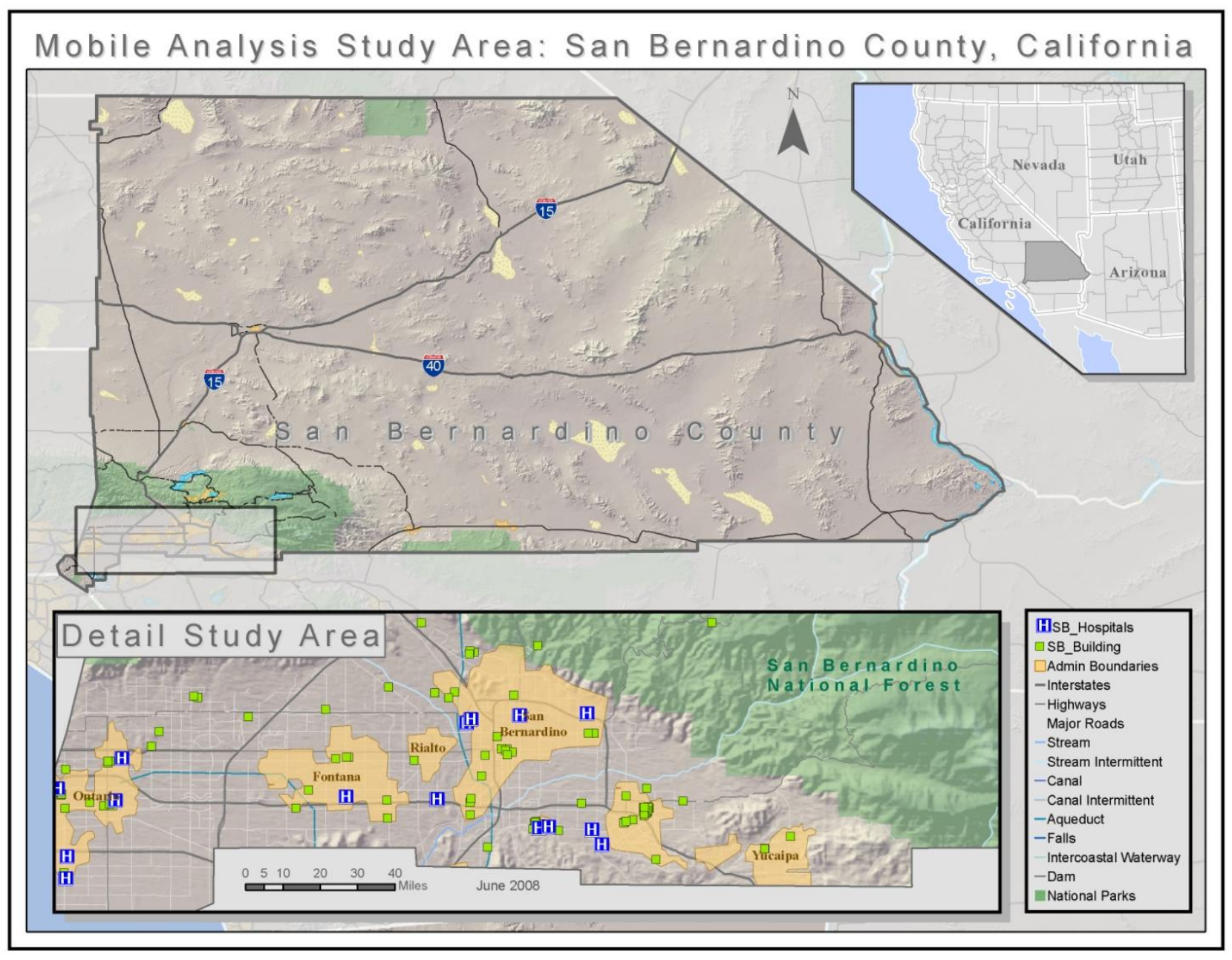

Figure 4.1 Project Study Area

\subsubsection{Database Considerations}

Since the mobile map cache would serve as the final geodatabase for this project, special considerations needed to be addressed. This included editing data on the mobile device, in particular the existing PREACT assessment layers and any subsequent analysis layers used in the project. "ArcGIS Mobile supports editing of data within multi-user (ArcSDE) Geodatabases only. Your mobile map can contain layers that reference other data sources, but if you wish to capture new features or update existing features, the layer's data source must be a feature class stored within an ArcSDE geodatabase." (ESRI, 2008(d)) Because of this, the project consists of two geodatabases, a file geodatabase for base data and an ArcSDE database for dynamic data layers. ArcSDE provides for a relational database 
management system which is necessary to allow for edits to data which will be dynamically updated from the field.

Planning for spatial analysis and model output included potential database considerations to allow for synchronization with the enterprise database. However, not all layers required a feature class as output from the analysis results. Some layers can be drawn locally as graphic layers and tables which are not stored in a database. With this in mind, ArcGIS Desktop was used to construct the resulting File and SDE geodatabases.

\subsubsection{Datum and Coordinate System}

The datum and coordinate system used for all data in this project are in the World Geodetic Coordinate System 1984 (WGS 84). All project data was projected in WGS 84, as it is the army standard and therefore a requirement of the client. The ArcGIS Mobile SDK also supports WGS 84 and requires no internal conversion to work with map drawing procedures on the device.

The use of WGS 84 also allows for the project to be more scalable as it is transitioned to a worldwide project. Since the WGS 84 datum is Earth centered, it can be used for geometric calculations throughout the world. The only area of concern about using this datum and coordinate system are areas located in close proximity to the poles, as distortions are the highest there.

\subsubsection{File and ArcSDE Geodatabases}

A file geodatabase was selected to serve as the project geodatabase for base layers. This was not only to replicate the PREACT design, but also to use the latest technology available from ESRI. All data collected from ESRI and other sources is stored in this single File Geodatabase as feature classes. These feature classes have also been appropriately grouped and stored in similar feature datasets which closely resemble the original PREACT database schema. Figure 4.2 shows these geodatabases and the collected data for use in the project.

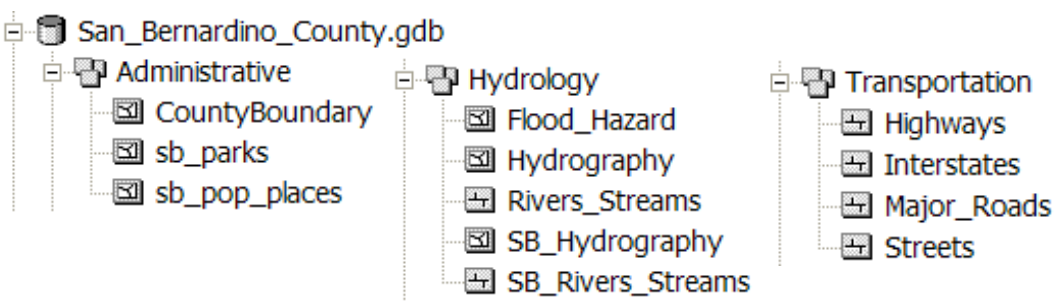

Figure 4.2 Project File Geodatabase

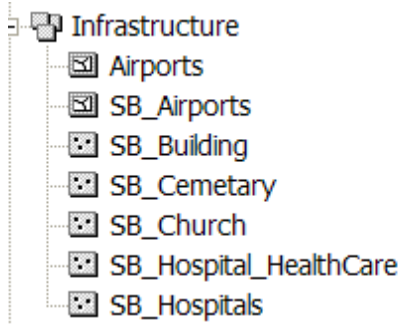


An ArcSDE geodatabase was also implemented which provides for feature classes which would be edited or synchronized from the mobile application to be stored. ArcSDE is required for any layers that are dynamically updated from mobile devices because of the editing and permission rules in the enterprise geodatabase. Figure 4.3 is a representation of this data for selected layers within the project area of concern which has been ported over from the ArcSDE database used in PREACT.

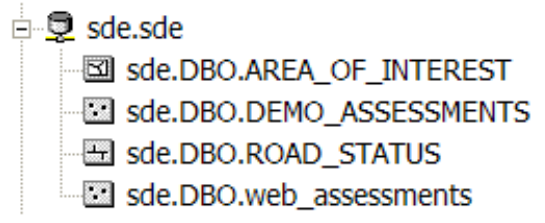

Figure 4.3 Project ArcSDE Database

\subsubsection{Mobile Map Cache}

A mobile map cache was created from a mobile data service containing all data with appropriate cartographic representations for mobile use. A mobile data service is created from an MXD, which contains the data for deployment and synchronization within the ArcGIS Mobile framework. A map cache serves as the local database instance, allowing for data access and display based on the mobile data service. This map cache is comprised of .bin files created within Arc Toolbox using an ArcGIS model developed specifically for extracting this data. This process creates a new .bin file for each layer within the MXD and stores it in the map cache in a proprietary format. The .bin files also hold the cartographic representations as they appear in the project MXD for display. The map cache deploys to the mobile devices with the mobile application to complete the mobile GIS application (Figure 4.4). The map cache references the base data to visually display, read, and write to while editing in the field. Upon synchronization from a field user the mobile map cache is updated with any edits to their local data through the mobile data services. This process also allows the user to post any edits they have made to the central database.

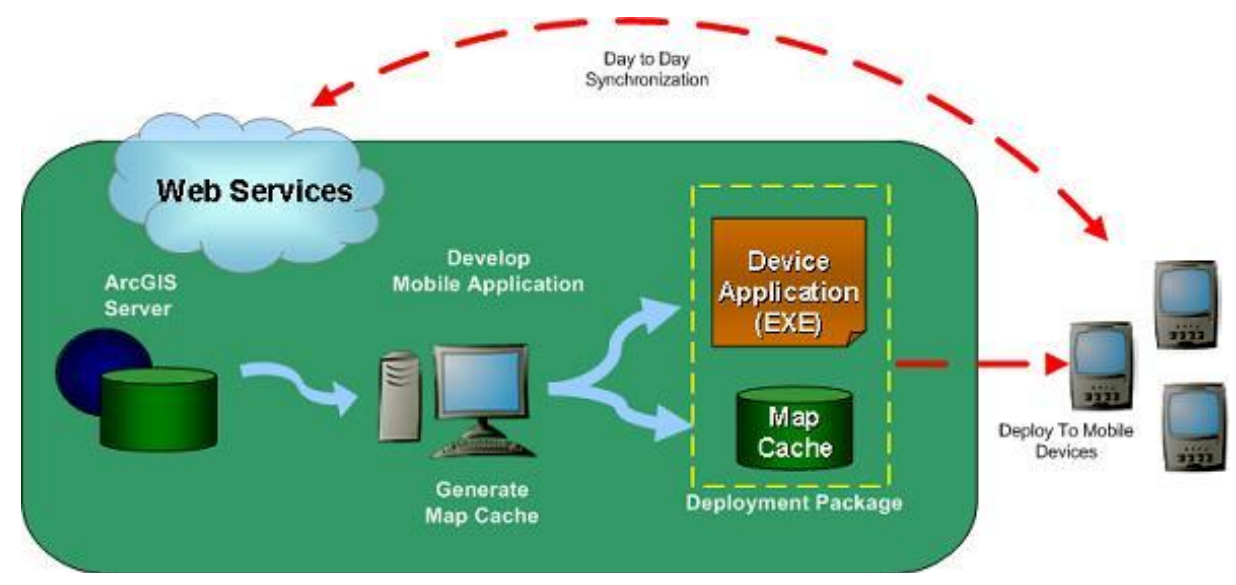


Figure 4.4 Deploying mobile applications (ESRI, 2008(f))

There was only one choice for a map cache in ArcGIS Mobile 9.2, which stored all the data deployed to the device. ArcGIS Mobile 9.3 introduced a new method that allows for the creation of two caches; one for base data, and the other for data which would be edited or synchronized with the enterprise database. This avoided a serious problem in which a synchronization call for the data on the mobile device would download all the map cache data, including infrequently updated base data. The PREACT developers created a custom process as a work around, synchronizing only the updated mobile and web assessment layers. Since this project must adhere to the existing PREACT application design, this cache design carried over to this project and the new system did not require creating two separate map caches, since the PREACT developers already handled this issue. Upon synchronization from the field, only the assessment data is updated to the mobile map cache. In the future this could potentially limit the updates of analysis layers and will require a re-examination of the process at that time.

\subsubsection{Cartography and Visualization}

Special considerations have been made when designing the mobile map, due to limitations within the Mobile SDK and hardware. A full list of these limitations can be found in Appendix C, which outlines the layers supported within the Mobile SDK. In general, the maps should be as basic as possible to be viewed on a small screen with symbology standards and simplified features. Figure 4.5 is a good example of the types of things that can be done to make better mobile maps for deployment. Not only is it difficult to visualize complicated features and symbologies, but it also slows the device down when map is redrawn. For example, the conventional map design on the left utilizes complex symbology for the water body and also incorporates building footprints. On the right, the complex symbologies are simplified with a basic hue fill and the building footprints are omitted to aid in reduced file size and processing, required to include them in the mobile map cache. 


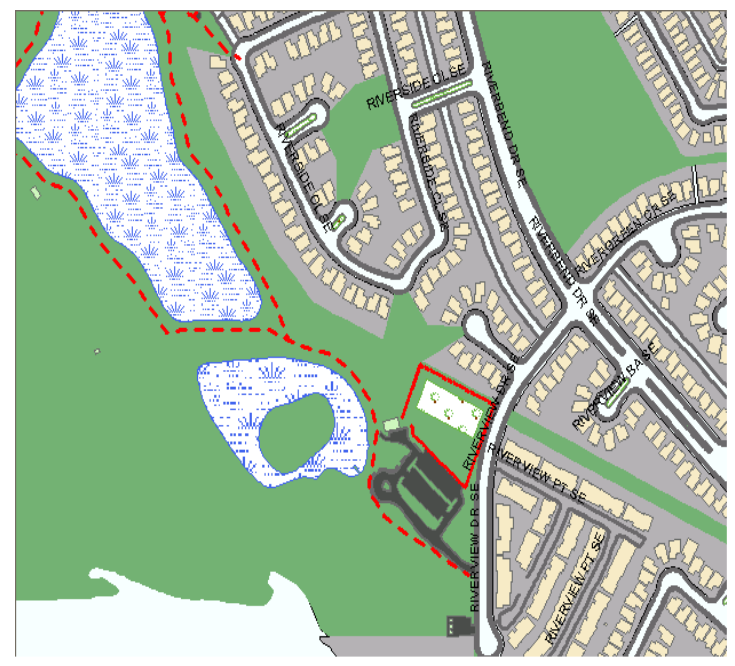

Conventional Design

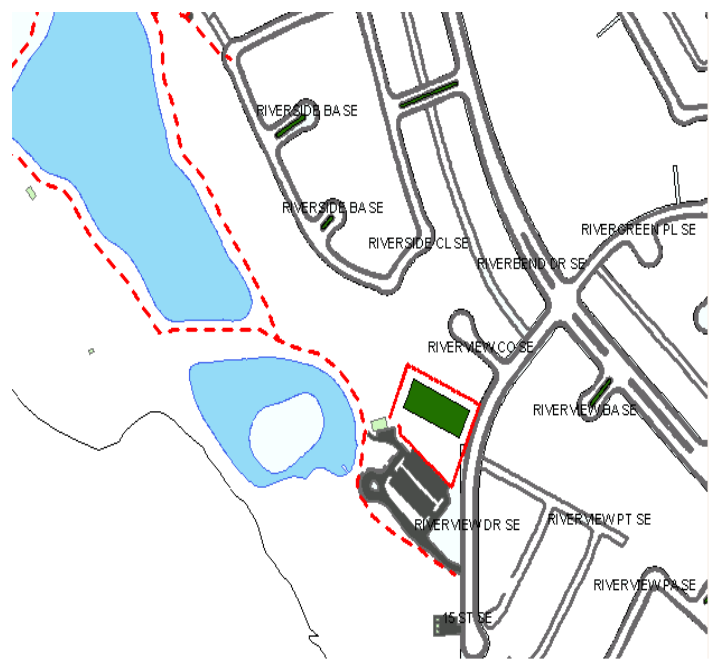

ArcGIS Mobile Design

Figure 4.5 Design standards for mobile maps (ESRI, 2008(c))

\subsubsection{Cartographic Limitations}

The mobile SDK does not support all layers and data types within ArcGIS Desktop. This is important in limiting corrupted or incomplete map caches. Supporting the output of a geoprocessing tool layer is of particular importance for this project. This enables updated geoprocessing outputs run by other users to be added to the mobile display through a synchronization process. Since there are multiple geoprocessing tasks available to web and desktop users within PREACT, it was possible to keep these outputs in an ArcSDE feature class and synchronize the results to the mobile client (ESRI, 2008(b)).

Another issue regarding ArcGIS Mobile mapping was the use of the Maplex labeling engine. It was necessary to rebuild all MXD's in ArcGIS Desktop 9.3 and ensure the Maplex label engine was not enabled.

\subsubsection{Design Environment}

When working with data and MXD's for use with mobile applications it is useful to replicate the ArcMap environment to the mobile resolution of the output device. Figure 4.6 shows an example of this process which allows for applying cartographic techniques in the desktop environment that closely match a mobile device screen. This allows for enhanced visualization once the data is on the mobile device, without the need for multiple tests on the device itself. 

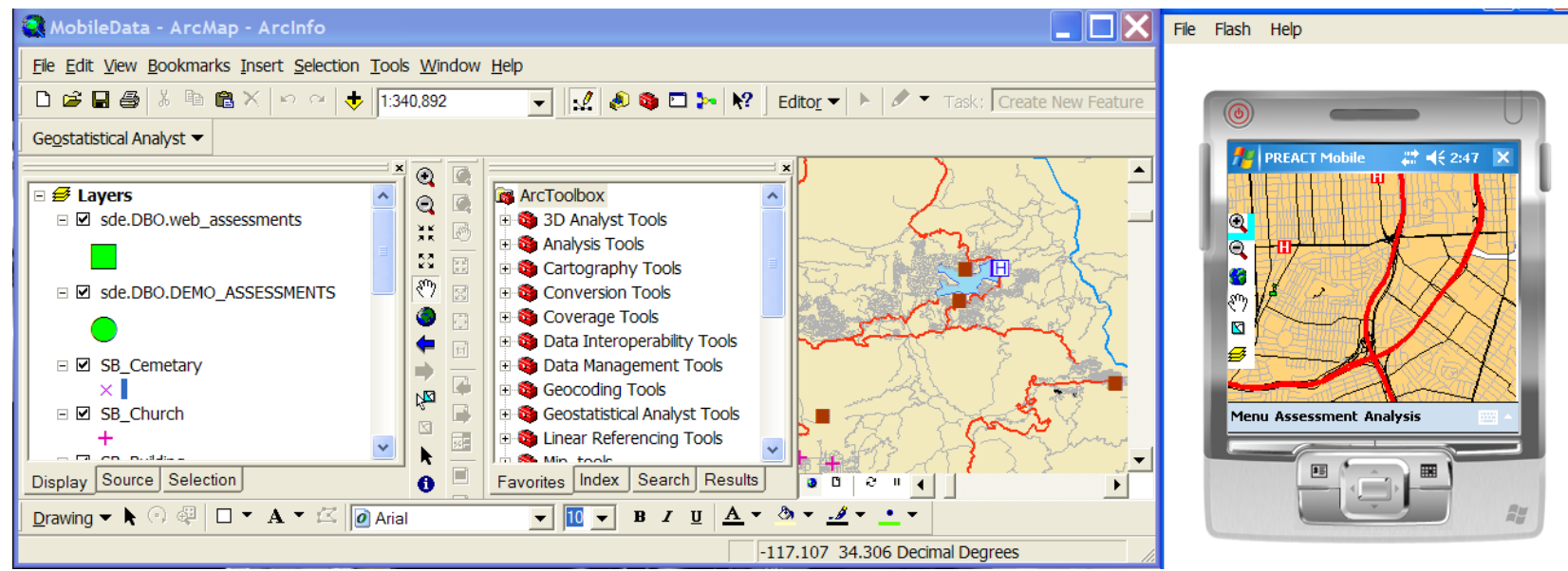

Figure 4.6 ArcMap Design Environment

\subsection{System Design}

\subsubsection{Development software}

The following software packages and products were used in the development cycle of this project. All of these software packages revolve around ArcGIS Server 9.3, the keystone in developing mobile applications through the ArcGIS Mobile Software Development Kit (SDK) available from ESRI.

ArcGIS Desktop $9.3-$

- ArcMap - This application was used to design and author the MXD's creating the mobile and geoprocessing services used in the project. ArcMap was used in the development phase and for updates to the MXD's.

- ArcCatalog - Data storage, creation, and manipulation of the project geodatabases were performed in ArcCatalog. The creation and management of web services were also handled within this ArcCatalog.

ArcGIS Server 9.3

- The basis for the PREACT system is an enterprise solution with centralized services offered by ESRI's ArcGIS Server 9.3. This software suite incorporated most of the development software used in developing the analysis tools for this project.

- ArcGIS Server allowed for the developers and administrators in the system to create and update the web services. Any changes to data or spatial models required an update to the services on the Server and were handled by the server administrator. 


\section{ArcGIS Mobile SDK}

- The Mobile SDK incorporates GIS functionality through development in the Visual Studio platform. The SDK allows for customization of basic mapping functionality, geometry functions and geoprocessing services. These tools were developed with the SDK for use on the handheld devices, customized to fit the needs of the end user.

- The SDK provided the building blocks to create and update the mobile software by the developers.

Map Cache Extractor

- A mobile toolset within ArcToolbox allows users to generate a map cache from a mobile data service, which is a new feature in ArcGIS Server 9.3. The extractor creates .bin files which store the data and attributes in feature classes and raster data, which resides on the central server. The combined .bin files, or map cache, is created to synchronize the data in a mobile data service, from the server. The mobile device leverages these .bin files to provide all the geographic information for field use.

Visual Studio 2008

- Visual Studio 2008 was used to develop the custom mobile application and to leverage the ArcGIS mobile SDK. These tools work with the .bin files (layers) to provide visual display and analysis.

Existing PREACT Mobile Software

- The Analysis tools will extend the existing PREACT architecture and software. TEC has supplied the base application which serves as a starting point for developing the analysis tools. This software development was performed in the Visual Studio environment, programmed in Visual Basic .NET languages, and was designed to work with the mobile and geoprocessing services.

- The existing software will handle the basic user input data and assessment collection process. This includes synchronization and replication of the existing PREACT assessment data.

- All data collected with this application synchronizes with the server and central geodatabase for dissemination to the entire enterprise system.

\subsection{Graphical User Interface}

The graphical user interface (GUI) was developed using the existing PREACT mobile design. This initial GUI contains all the mapping and tool buttons, allowing user interaction with the system. The following are the key components in the PREACT mobile system allowing for this user interaction. They include the map interaction, application settings, GPS settings, assessment collection interface, and data synchronization operations shown in Figure 4.7. 


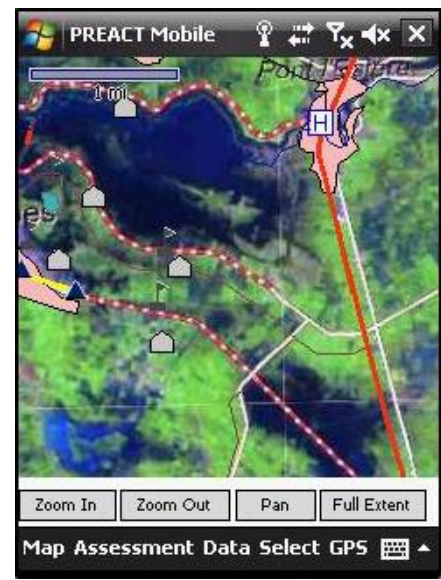

Figure 4.7 PREACT Mobile GUI

Adding the analysis tools requires an additional toolbar button to access the various tools and to activate any additional dialogs for user input. The analysis tool functionality will require the user to select the layers from which they will run the analysis and model parameters necessary to complete the required inputs. Each new analysis tool will require a dialog interface to guide the user through the analysis process. The final analysis output will display on the main map as an output graphic layer for visual display or as a table of features from existing layers.

\subsubsection{User Interface Design}

The existing PREACT mobile user interface is not adequate to support new tools with all the capabilities of the existing application. Based on observations from the field during testing, a redesigned interface was also required which was more intuitive and also incorporated the necessary buttons for the new analysis tools. The left image in figure 4.8 shows the existing PREACT mobile GUI, housing all main tool bar functions. The map control tools are cluttered and the text driven buttons could be more intuitive. The right interface design shows the redesigned GUI, which condenses the functionality and tools into two groups which contain all the tools available in the application. The menu button contains the map and GPS controls, while the tools button contains all the tools available to interact with the mobile map and data. The map controls have also been changed to pictures of the tools they represent, which is standard in most GIS software packages and web applications (e.g. Zoom In tool is a magnifying glass). 

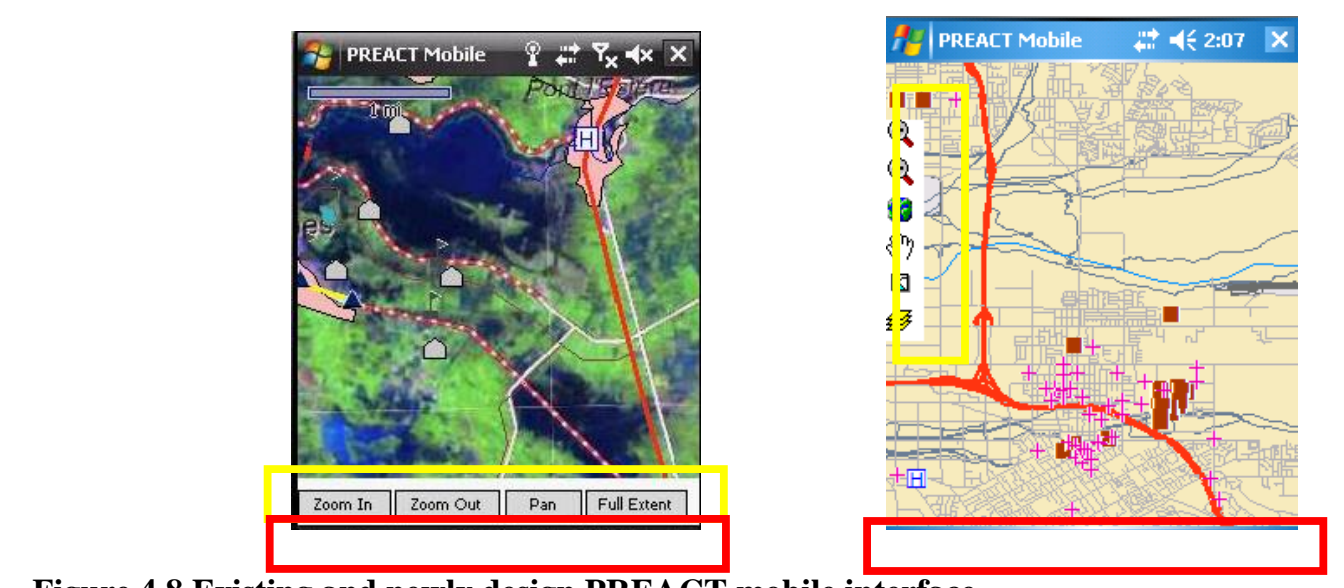

Figure 4.8 Existing and newly design PREACT mobile interface

Some of these redesign decisions were based on the direction of other popular mobile applications, such as Google Maps Mobile and Microsoft Windows Live Search Mobile. A common theme across these applications is a main toolbar consisting of one or two command buttons and integration with external buttons to interact with the map. Figure 4.9 shows the simple, intuitive interfaces which many mobile developers have adopted.

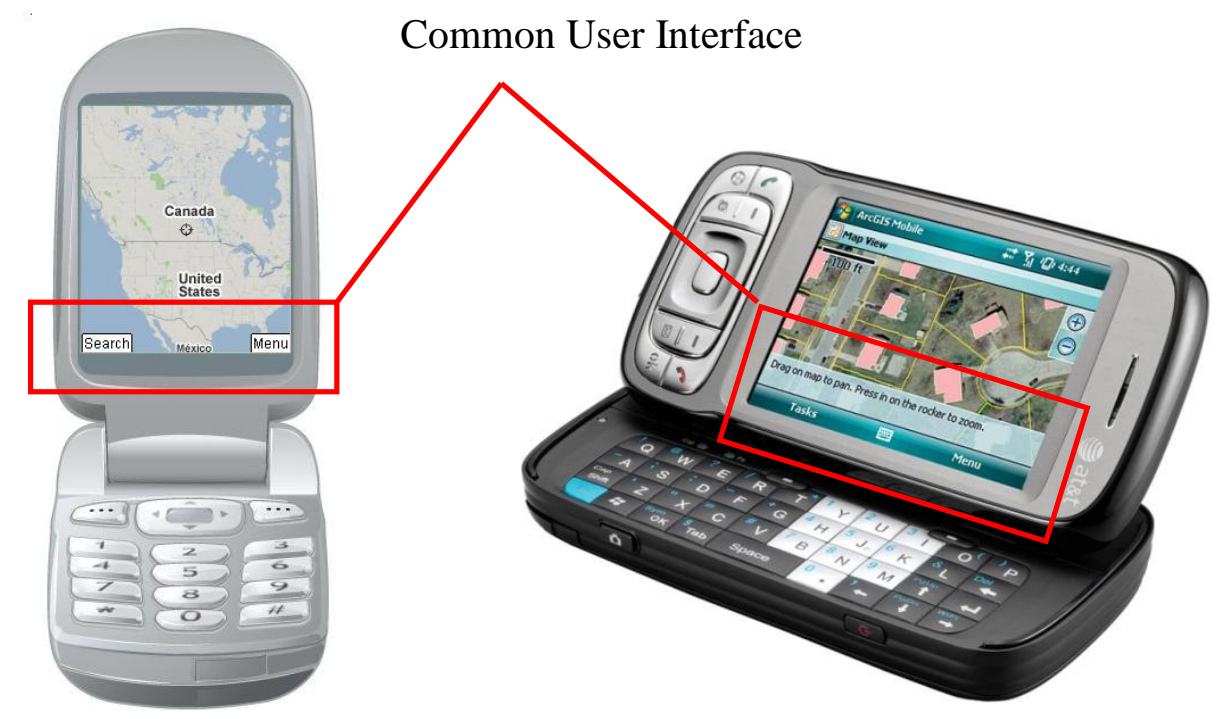

Figure 4.9 Common Mobile Mapping Interfaces Showing User Interface Designs (Google, 2008) (ESRI, 2008)

Based on these findings it was necessary to research ways to better implement the functionality within the PREACT mobile application. Since the PREACT application offers more functionality than simple mapping applications, changes were necessary to aid 
in usability and to minimize training. One key change was a move to graphic-based buttons, as opposed to strictly relying on text-based menus and buttons.

\subsection{System Architecture}

The system architecture has three key components desktop GIS, server GIS, and mobile GIS that revolve around a central database. Each component works in conjunction with ESRI's ArcGIS 9.3 software suite, which encompassed all components of the system design (Figure 4.10).

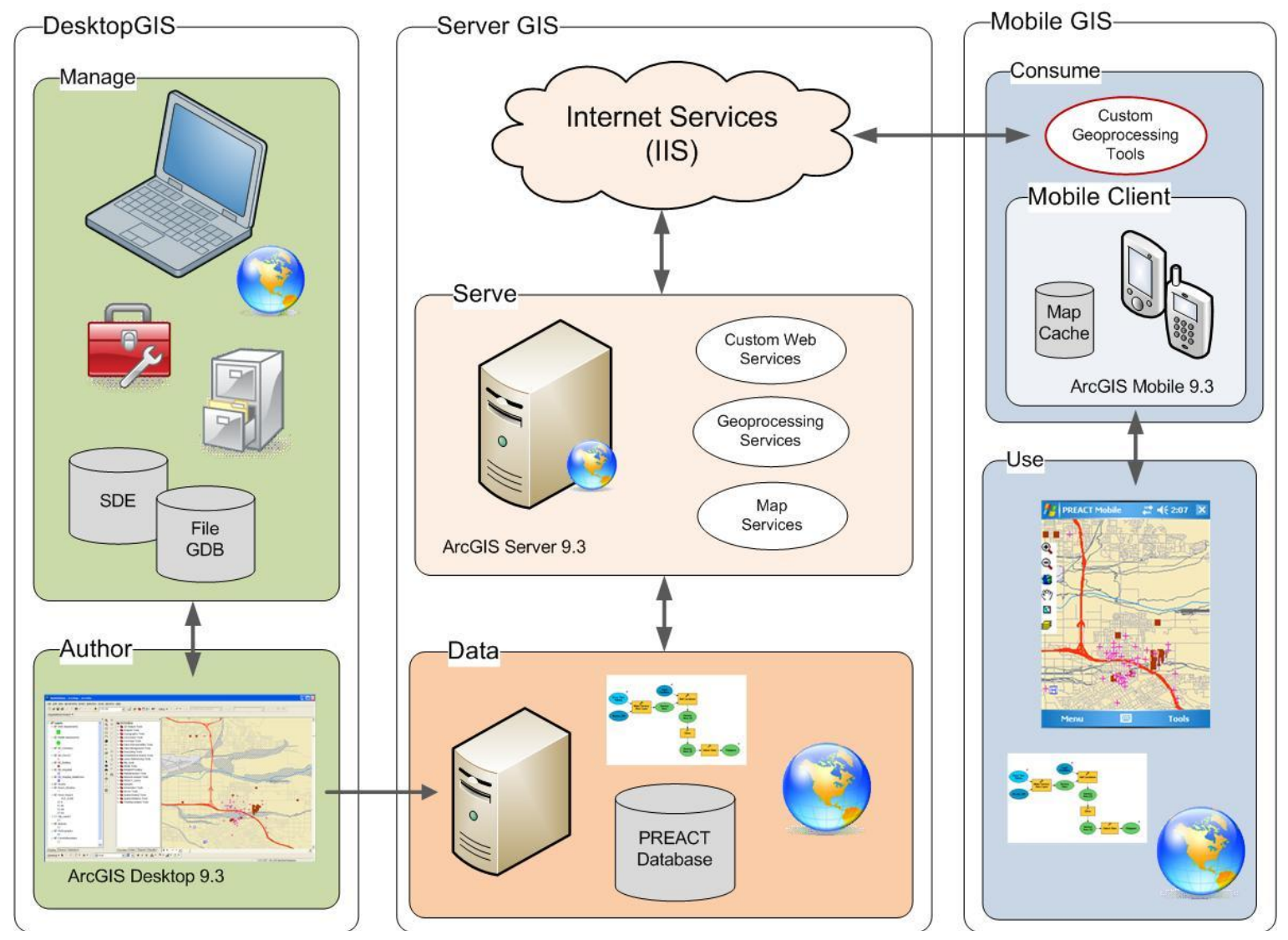

Figure 4.10 Final System Architecture

\subsubsection{Desktop GIS}

The desktop GIS component consists of ArcMap, ArcCatalog, and ArcToolbox, which serve as the management and partial development software technologies. Within ArcMap, the MXD's were developed for use on the mobile applications. Spatial models were created and managed within ArcToolbox which will be deployed as geoprocessing services. All data management and creation was done within ArcCatalog. 


\subsubsection{Server GIS}

The server GIS components were developed using ArcGIS Server 9.3. ArcGIS Server provides connectivity to geographic information through web services, which the PREACT system utilized. The GIS server provides a mobile SDK used for mobile application development. Within ArcGIS Server, all map and geoprocessing services are created and served through web services, which are consumed by the end user, in this case a mobile client. The server also hosts the geoprocessing services, which run the spatial models and return the results to the mobile device.

\subsubsection{Mobile GIS}

The final component is the mobile GIS application. "ArcGIS Mobile is a task-driven mobile application for Windows Mobile devices that uses a web service architecture to synchronize information between the field and office. ArcGIS Mobile also includes an SDK for building very focused applications that run on Windows Mobile devices, Windows CE .NET devices, and Windows Tablet PC devices." (ESRI, 2008(g)) This framework allows access to geospatial data, as well as services which enable enhanced functionality on the mobile platform. This component is the host for triggering geoprocessing tasks residing on the server, as well as geometry-driven spatial analytical tools for use while in the field. 


\section{System Implementation}

A general implementation approach was constructed with the completed system and database design. Development utilized a spiral development methodology, starting with the implementation of a sample application for proof of concept. This application is the foundation for the final prototype system to be developed and focused on the services required to access and run a geoprocessing task residing on the central ArcGIS Server discrete from the PREACT baseline. The sample application had a complete data set and deployable application and required the correct implementation of the custom web service and geoprocessing services to be configured to work on the test server.

With the sample application functioning, the modified services could be extended for use in the existing PREACT application. The following implementation documentation outlines this process.

\subsection{Initial Proof of Concept}

It was determined, through initial research and collaboration with ESRI, that triggering geoprocessing services from a mobile application is possible with additional communication protocols between the mobile device and the server. Project implementation began with a series of sample applications which implemented this process.

\subsection{Modified Sample Mobile Geoprocessing Application}

Since there is no out-of-the-box solution for incorporating geoprocessing tasks into a mobile application, a sample mobile application from ESRI was first implemented. This sample mobile application was based on providing geoprocessing services to the mobile client when connected to the server. To achieve this, a custom web service was necessary to pass the needed information from the mobile device to the geoprocessing service. This was due to the .NET compact frameworks incompatibility to work with ESRI's geoprocessing services. Figure 5.1 illustrates how the custom web service communicates with the geoprocessing service to pass the input parameters and objects to run the task, and then to pass the output back to the mobile device. This process also demonstrates the modifications to the initial architecture that enables a communication protocol to broker communication between the .NET Compact Framework and ESRI's Geoprocessing services framework (shown as a red dotted line). 


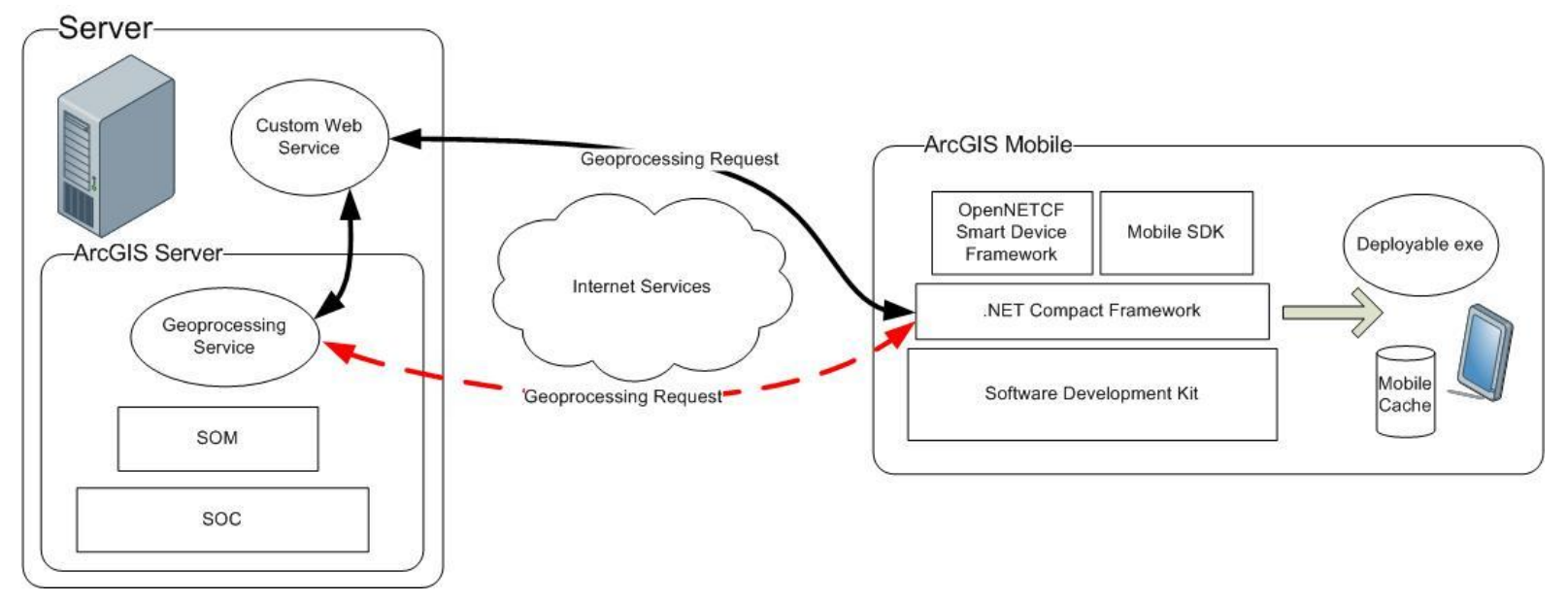

Figure 5.1 Custom Mobile Application Architecture

\subsubsection{ESRI Sample Data}

ESRI supplied data for use in the sample application. This data included a network dataset; a street network topology layer (Streets_ND) for the San Francisco, California, area (shown in Figure 5.2).
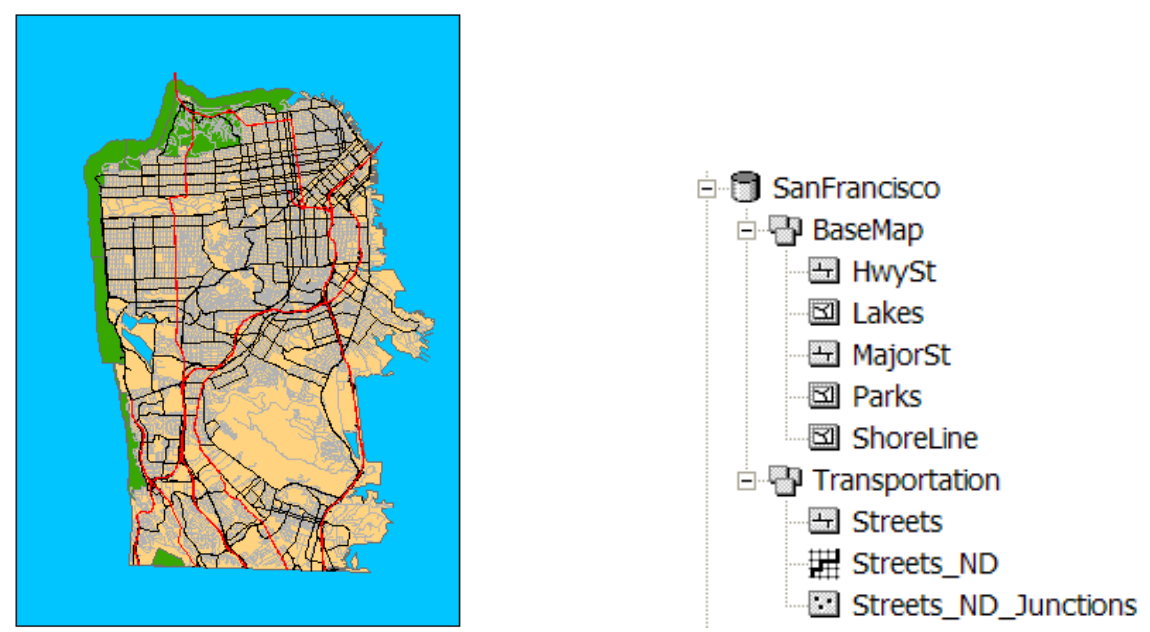

Figure 5.2 ESRI Sample Application Data 


\subsubsection{Drive Time Polygon Geoprocessing Service}

This geoprocessing service is a drive time calculation model which leverages ArcGIS Network Analyst. The sample ESRI model receives the input point from the mobile application and performs a drive time calculation based on a road network. The server leverages Network Analyst and the streets network dataset to create an output polygon showing the maximum distance one could travel, based on an input time. This polygon is then passed back to the mobile client and displayed on their map.

A geoprocessing service is required to leverage the model through the hand held application. The model and output feature class is added to an MXD as a geoprocessing tool layer. The MXD is then published as a map service that contains a geoprocessing service. This is done through the ArcGIS Server Manager or ArcCatalog.

\subsubsection{Custom Web Service}

As previously stated, an inherent problem in the .NET compact framework is its inability to communicate with ESRI's proprietary geoprocessing service. There are several workarounds for this issue. However, only one of them was considered to be reasonable for this project. One option, as previously done by the ESRI developers, is to modify the geoprocessing service core framework to allow for the .NET compact framework to communicate with it. This requires proprietary source code and knowledge of ArcGIS Server services and therefore, outside of the project scope. Due to the complexity of the other options it was decided, through collaboration with ESRI, to implement a custom web service that provides a communication protocol to the geoprocessing services. The sample application from ESRI uses this custom web service framework to communicate between the mobile application and geoprocessing service and provides an example of how to design and implement the custom web service.

This web service was configured as a standalone web service within Visual Studio 2008 and is served through standard internet services and acts as an intermediary for the application and the geoprocessing service, both residing on the same server. All inputs which need to be passed to the geoprocessing service are first passed through the custom web service. This creates new challenges for developers, including how to handle ESRI geometry types (points, lines, polygons) in an environment which does not inherently recognize ESRI geometry types. For points, this process is relatively straight forward, as an $\mathrm{X}-\mathrm{Y}$ coordinate pair can be passed through the web service. However, this is more challenging for a polygon input, as a series of points need to be passed as a coordinate collection and properly assembled for input into the geoprocessing service.

\subsubsection{Geoprocessing Sample Application}

After the custom web service and geoprocessing services were configured, the sample mobile application code was compiled and deployed for testing. The resulting sample application demonstrated the process for implementing connected spatial analysis tools by accessing the custom web service that was able to interact with the ArcGIS Server. This 
process proved to be more difficult than expected and required a complicated programming and architecture setup to function properly. Such difficulties include the custom web service integration and configuring the necessary permissions and parameters to work with the test server. Since the web service is outside of the typical ESRI architecture for implementing mobile applications, there were few resources on how to properly implement a service which handles communication between ESRI's proprietary geoprocessing service and the .NET Compact Framework. ESRI's mobile development team assisted in this process and aided in configuring Internet Information Services (IIS) for the test server used in development.

Figure 5.3 shows the resulting sample application and the output polygon generated from the geoprocessing service. The polygon shows a one minute drive time from the central point, which was interactively selected on the map.
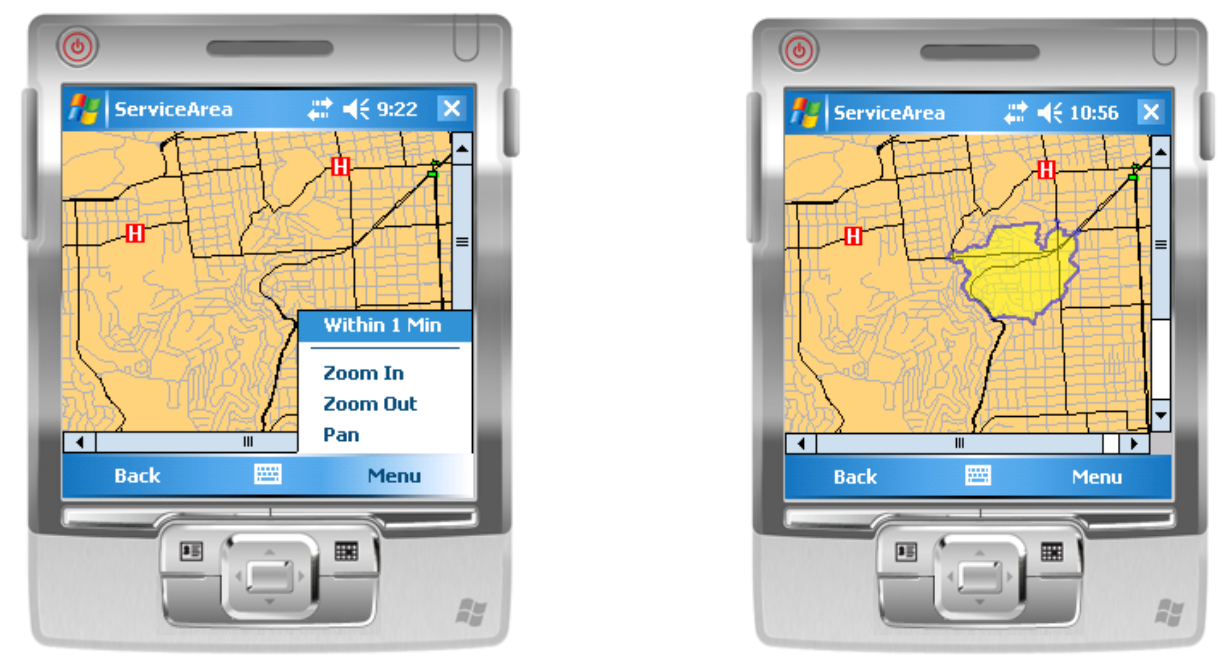

Figure 5.3 Modified ESRI Sample Application Interface and Model Output

\subsection{PREACT Pre-Prototype Implementation}

Once the initial sample application was successfully configured and working on the local server, it was implemented within the PREACT architecture. This required the code to be converted from C\# to VB.NET and a new command button control to be incorporated in the existing PREACT mobile application. The initial drive time sample data and services were used and deployed to the mobile device for this development task.

\subsubsection{Code Conversion}

The following VB.NET code is the final output from the original sample C\# code supplied by ESRI. This code creates a threading process which bundles the input $\mathrm{X}-\mathrm{Y}$ coordinate pair from a map click procedure and passes it to the custom web service which communicates with the geoprocessing service on the ArcGIS Server. 


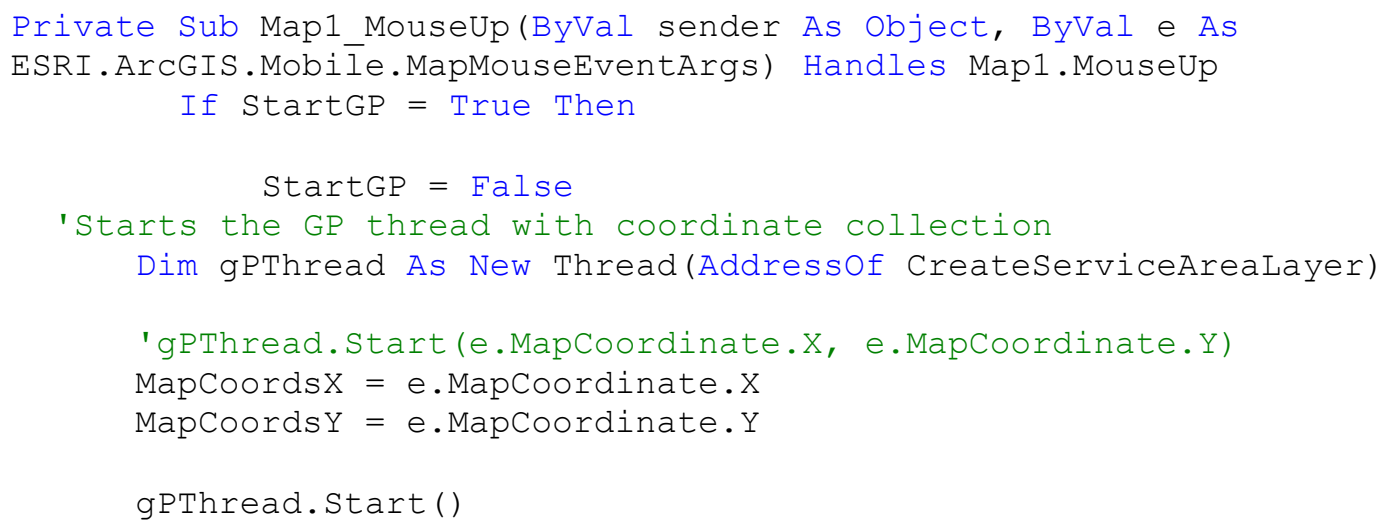

The CreateServiceAreaLayer() function handles the coordinate collection, which is sent to the web service and handles the drawing of the output polygon. In this case the returned coordinate collection from the Drive Time Polygon geoprocessing service is contructed (mPolygon) on the mobile device, added to the map cache graphic layer, and rendered on the map display. This is done in the following code:

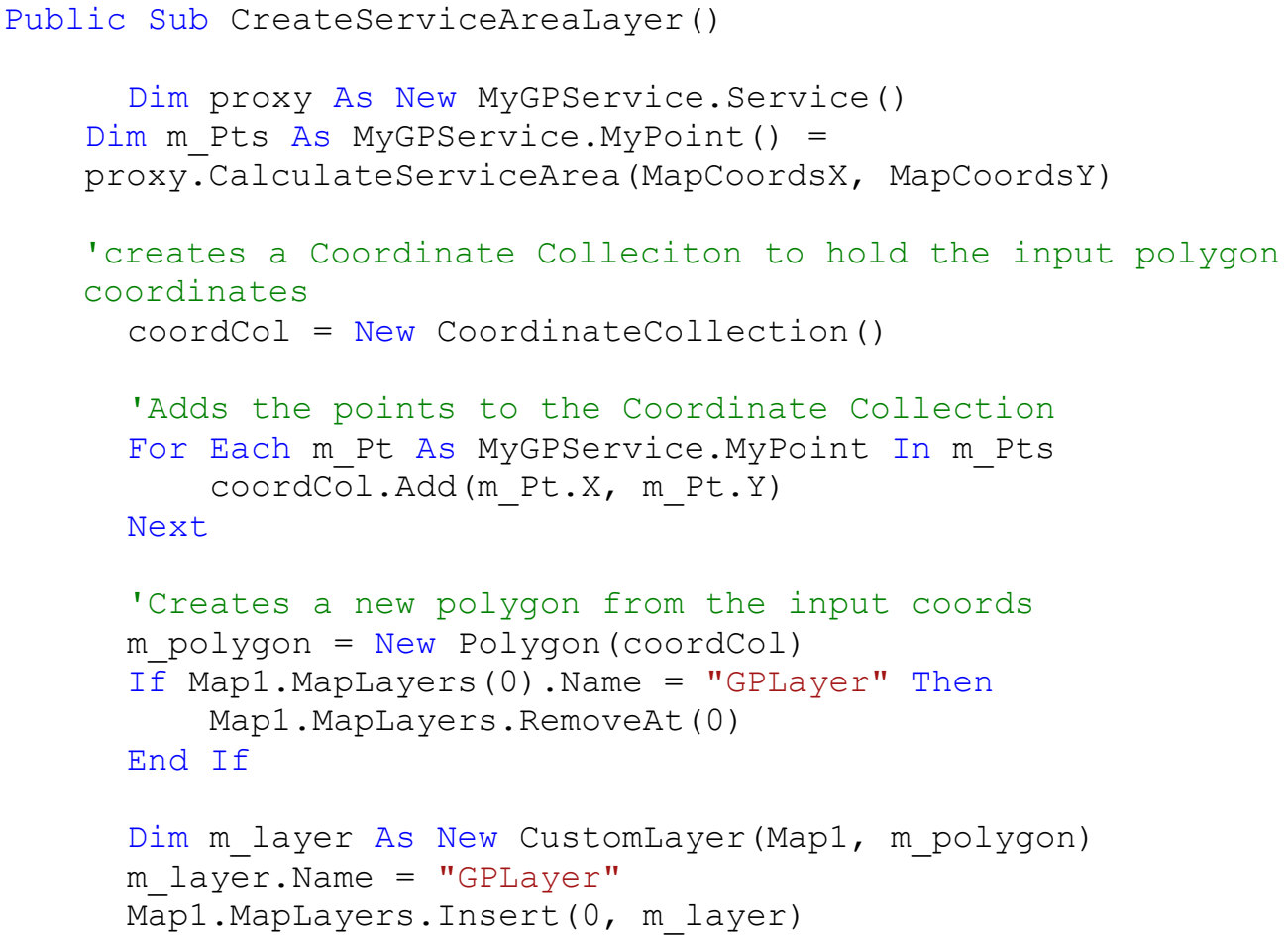

End Sub 


\subsubsection{PREACT Pre-prototype Deployment}

After the code conversion was complete, the PREACT source code was compiled and deployed using the existing San Francisco ESRI sample application map cache. The tool was tested to work within the PREACT application, which was successful. Figure 5.4 shows the output of a drive time polygon based on the sample geoprocessing service.

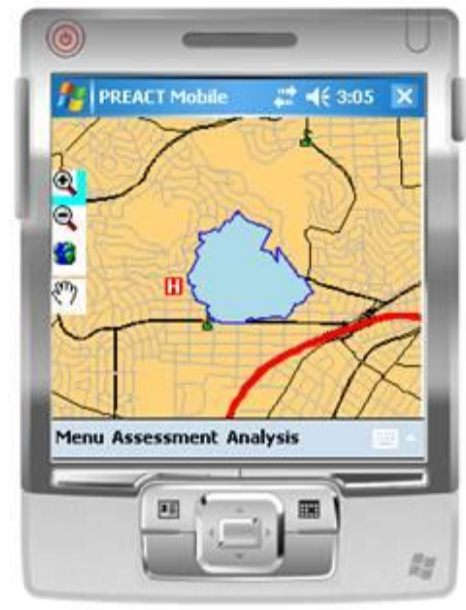

Figure 5.4 Sample Geoprocessing task runnning in PREACT Mobile

\subsection{Prototype Mobile GIS Analysis Tool Implementation}

The final proof of concept step was to integrate the pre-prototype framework with a custom geoprocessing service already available in the web version of PREACT. Several geoprocessing tasks were analyzed for possible inclusion and, with guidance from the client, it was decided to implement the population count geoprocessing service. This geoprocessing task is available from ESRI, located on their sample services server (http://sampleserver1.arcgisonline.com). The ability to analyze basic population counts adds a great deal of detail to assessments and is a good foundation analysis for inclusion into the PREACT framework. As the framework evolves and models mature, the population count is one that can provide inputs to many different analyses.

\subsubsection{Geospatial Data}

The data used for this final prototype was developed to work with the field-collected project data and the worldwide available LandScan data from Oak Ridge National Laboratory (ORNL). The LandScan data resides on the ESRI server with the population count geoprocessing service. Figure 5.5 shows a sample of the grid-based dataset, which has basic population counts in raster format. Each pixel in the raster represents a value for the estimated population. "The LandScan ${ }^{\mathrm{TM}}$ Dataset comprises a worldwide population 
database compiled on a 30" X 30" latitude/longitude grid. Census counts (at sub-national level) were apportioned to each grid cell based on likelihood coefficients, which are based on proximity to roads, slope, land cover, nighttime lights, and other information" (Laboratory, 2007).

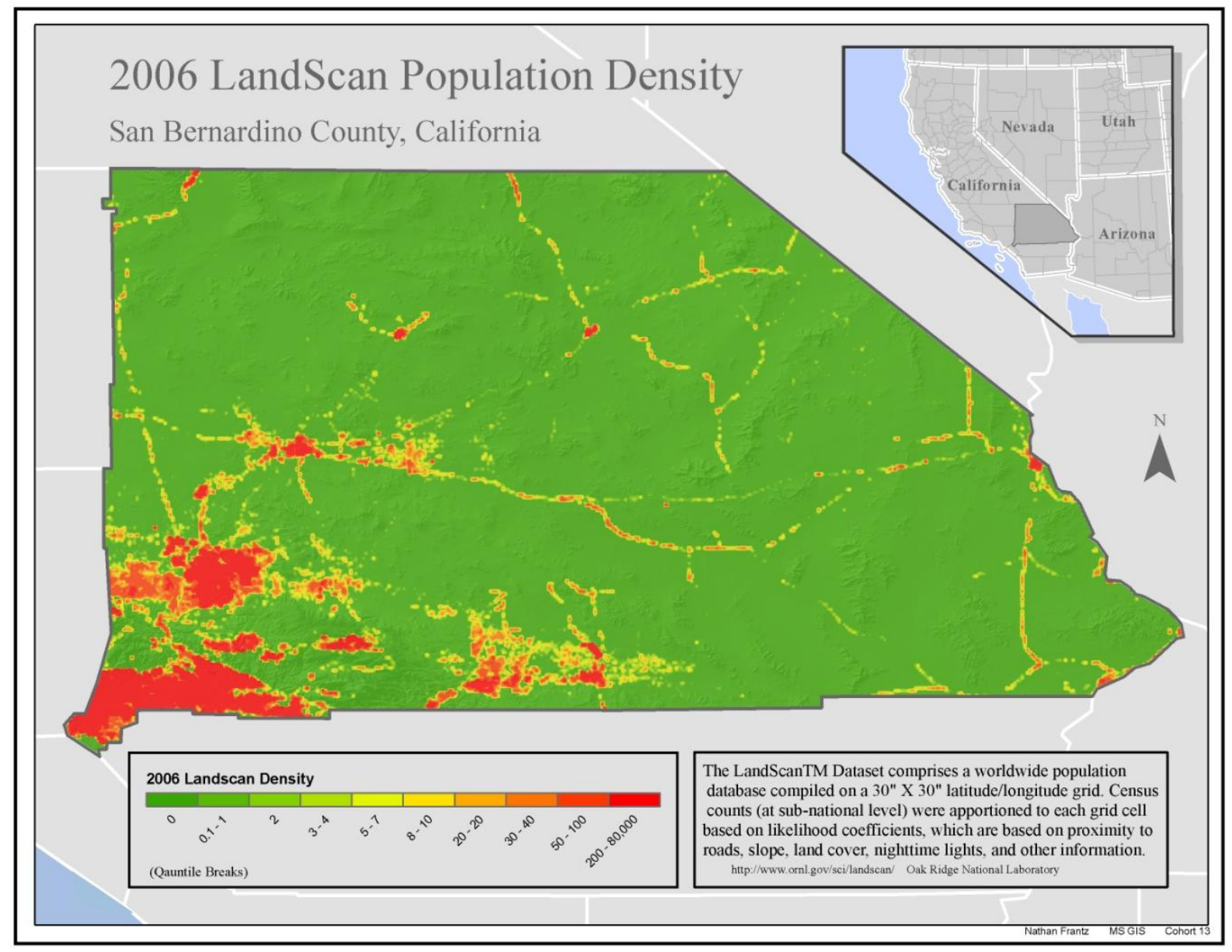

Figure 5.5 Sample LANDSCAN data for San Bernardino County

\subsubsection{Geoprocessing Service}

Configuration and deployment of the prototype sample geoprocessing service was done locally on the test server. The geoprocessing service used in the prototype application is hosted on ESRI's sample server, requiring the web service to simply point to the sample server to access it. The sample server from ESRI hosts multiple geoprocessing and data services which are available to any ArcGIS user. It was initially thought that the population count geoprocessing service could be recreated and hosted locally, but after an 
attempt to recreate it the sample server geoprocessing task was used. The speed at which the sample server service ran was far superior to any locally created service which sought to accomplish the same thing. On the down side, this geoprocessing service and data are not hosted locally and could be removed or changed, rendering their use ineffective.

Therefore, they are simply referenced for this prototype application and cannot be relied on for use in the PREACT system.

The population summary geoprocessing service configures based on a zonal summary of the LandScan data set. The model (Figure 5.6) takes input polygon and LandScan data set and calculates a statistical summary of population and area parameters based on an overlay function. The output is a string, or series of records containing that data.

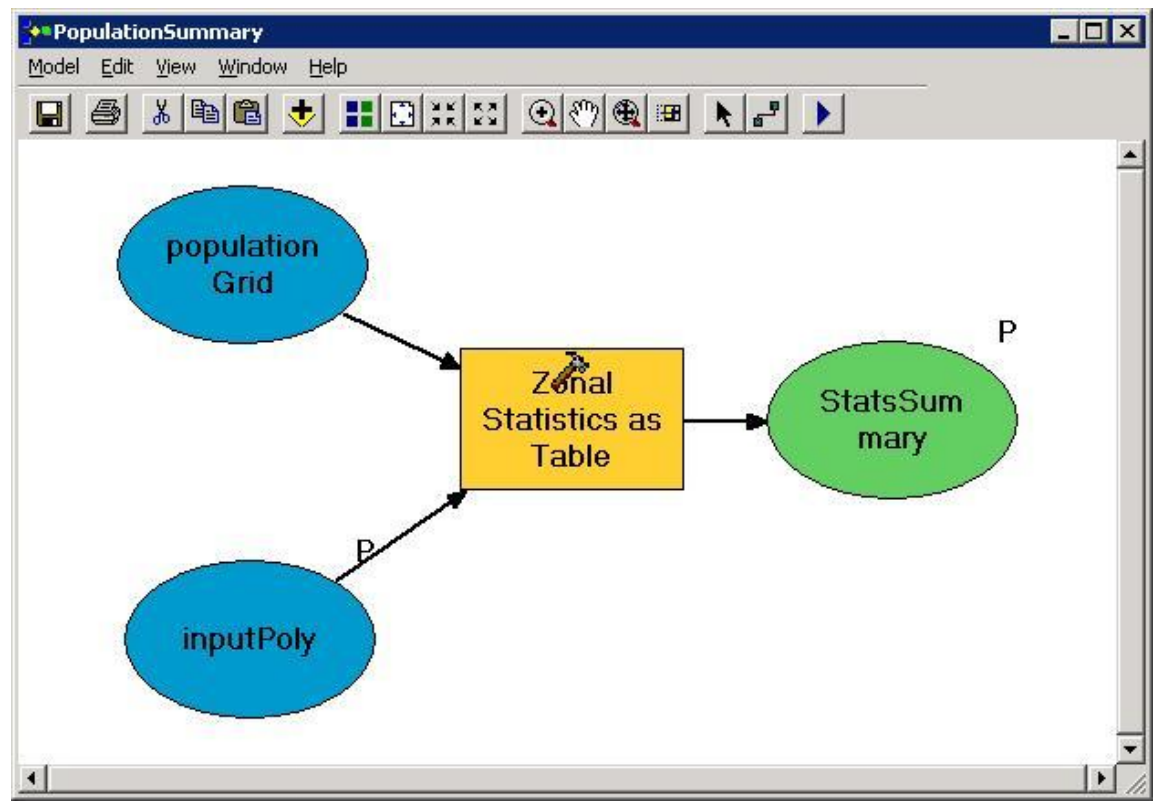

Figure 5.6 Population Summary Model (ESRI 2008)

The input and output parameters are typically developed from the internal spatial model. Since this geoprocessing service already exists, it was necessary to look at the Representational State Transfer (REST) resource to determine required input parameters (Figure 5.7). The custom web service is constructed with these service parameters to handle the appropriate geometry and attribute definitions. 


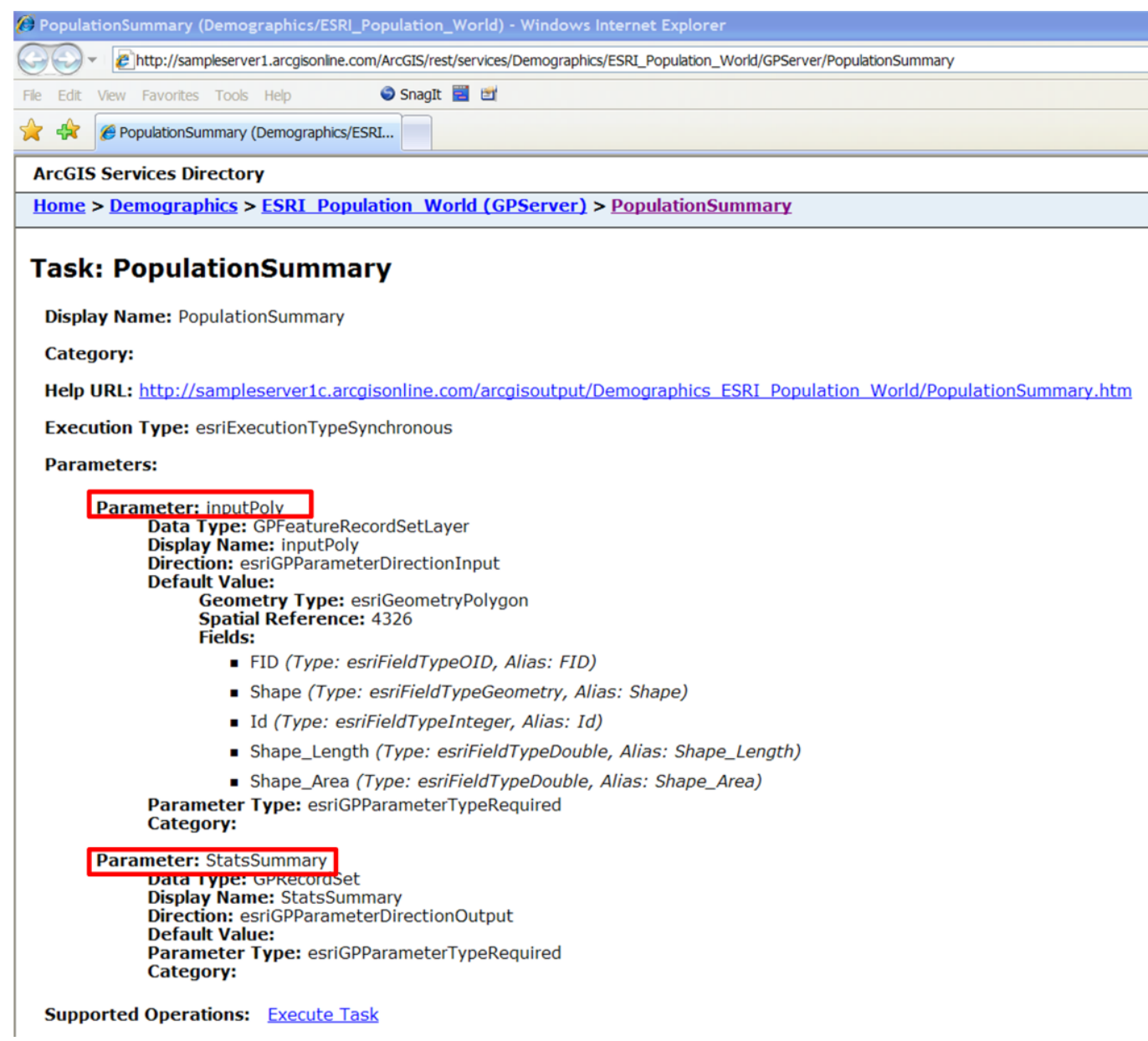

Figure 5.7 Population Summary geoprocessing service REST resource (ESRI, 2008(a))

The REST service description outlines the input and output parameters for the geoprocessing service. The service is looking for an input GPFeatureRecordSetLayer as an inputPoly or input polygon feature. The GPFeatureRecordSetLayer is internal to the ESRI geoprocessing service and constructs the input feature geometry as well as the attributes of the feature.

The output type is a GPRecordSet data type, returned from the geoprocessing service after the model has run. The GPRecordSet holds the records and values for the output statistical summary from the population summary model. The table output values display on the mobile device as the final output.

\subsubsection{Custom Web Service}

Based on the required geoprocessing input parameters, the custom web service was configured to accept an input polygon and return a string. In the sample application, the 
geoprocessing service needed an input point which would only pass a single X, Y coordinate pair. To enable a polygon geometry type, a user-defined coordinate collection is passed to the custom web service, where its geometry is reconstructed as input for the geoprocessing service. The coordinate collection is created from four input points specified by the user on the mobile device. This polygon input parameter must be constructed within the custom web service for the geoprocessing service to recognize these input parameters, since the web service will not recognize ESRI geometry types.

This stage of development was assisted by ESRI personnel, who constructed an input polygon without the use of the internal geometry types of the ArcGIS software suite. This core polygon geometry was then passed to the processing service as input. The following code was an example of how this was done using a PolygonN object and referencing a ring and point construction function to recreate the polygon geometry and its attributes. (See Appendix D for full code reference) The ring defines the edges or connections between points and a ring array defines each edge of the polygons based on the input coordinate pairs.

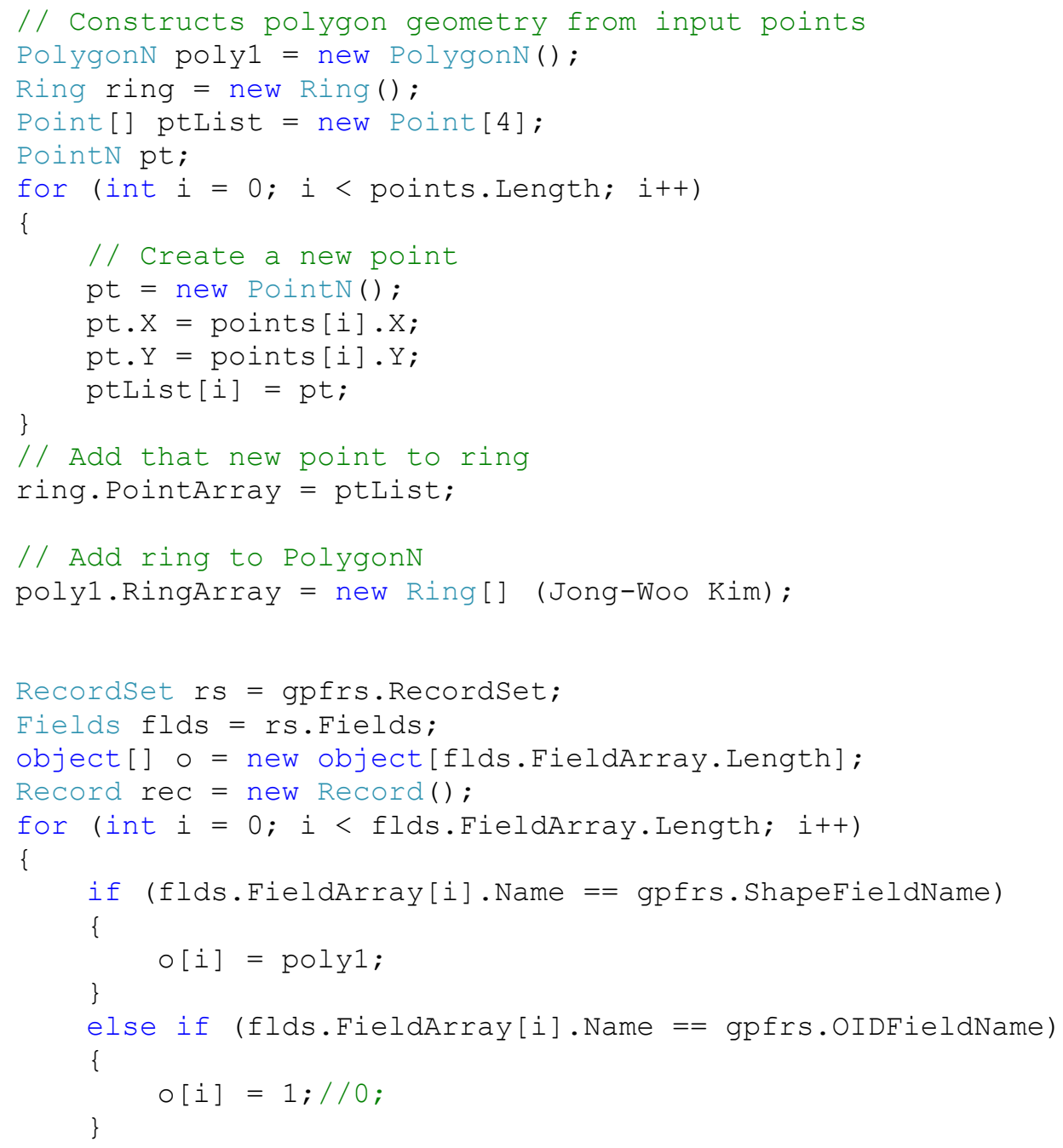




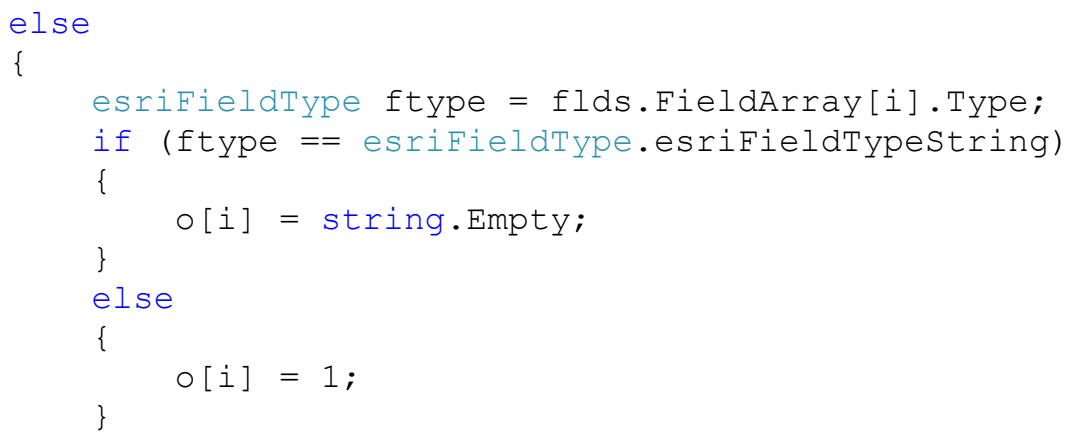

\subsubsection{Mobile Analysis Tool Software Development and Deployment}

Along with the addition of a new command for the population count analysis tool (Figure 5.8), user feedback for entering the input polygon needed to be developed. Since the input was a polygon, a map draw function had to be integrated to provide instant feedback as the user-added the polygon to the map.

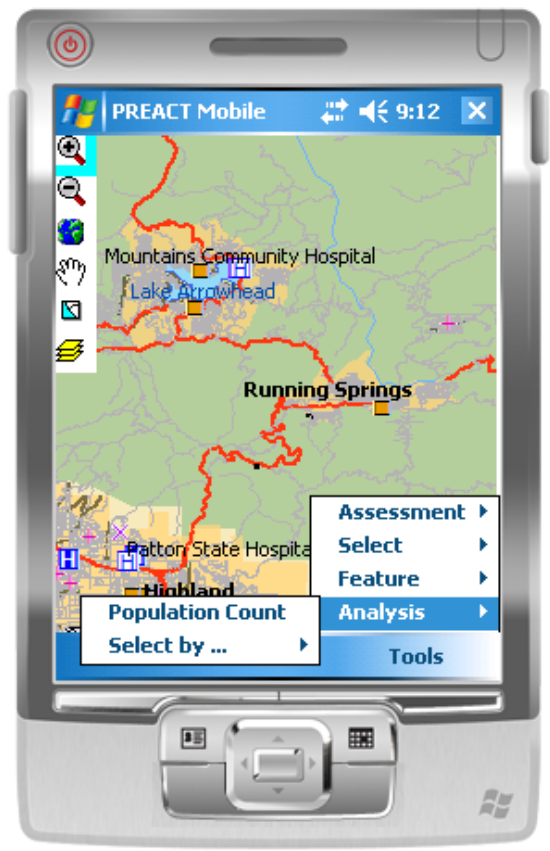

Figure 5.8 Project Interface and Analysis Tool Control

The user can begin entering points by clicking on the analysis tool button on the map (Figure 5.9). Each click creates a new point graphic where the user clicks and a line graphic is drawn between each point. Once the user inputs a fourth point, the coordinate 
collection is automatically completed and the feature is drawn as a closed area to represent the input polygon.

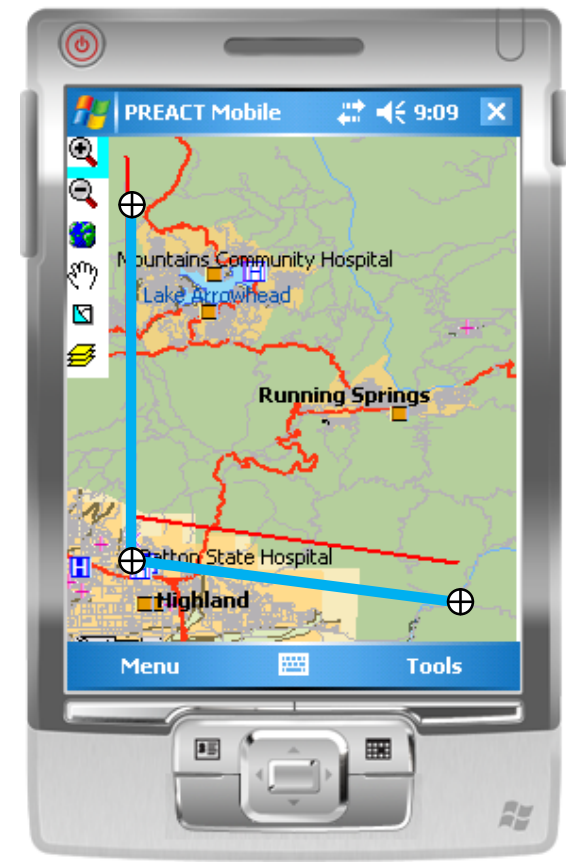

Figure 5.9 User Feedback for Polygon Creation

Along with adding a map graphic for user feedback, the input points are being recorded and stored as an $\mathrm{X}, \mathrm{Y}$ coordinate collection. Once the coordinate collection count reaches four, the coordinates are passed to the custom web service for input as the input polygon for the model.

The following code snippet shows the coordinate (m_coordinateCollection) being constructed with the input map clicks (see Appendix E for full code reference). When the coordinate collection count is equal to four, the coordinate collection is then passed to the web service through a threading process on the device.

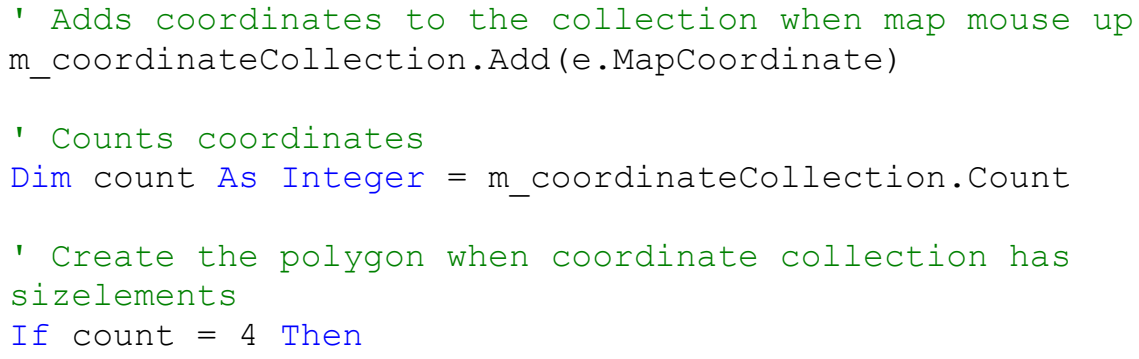




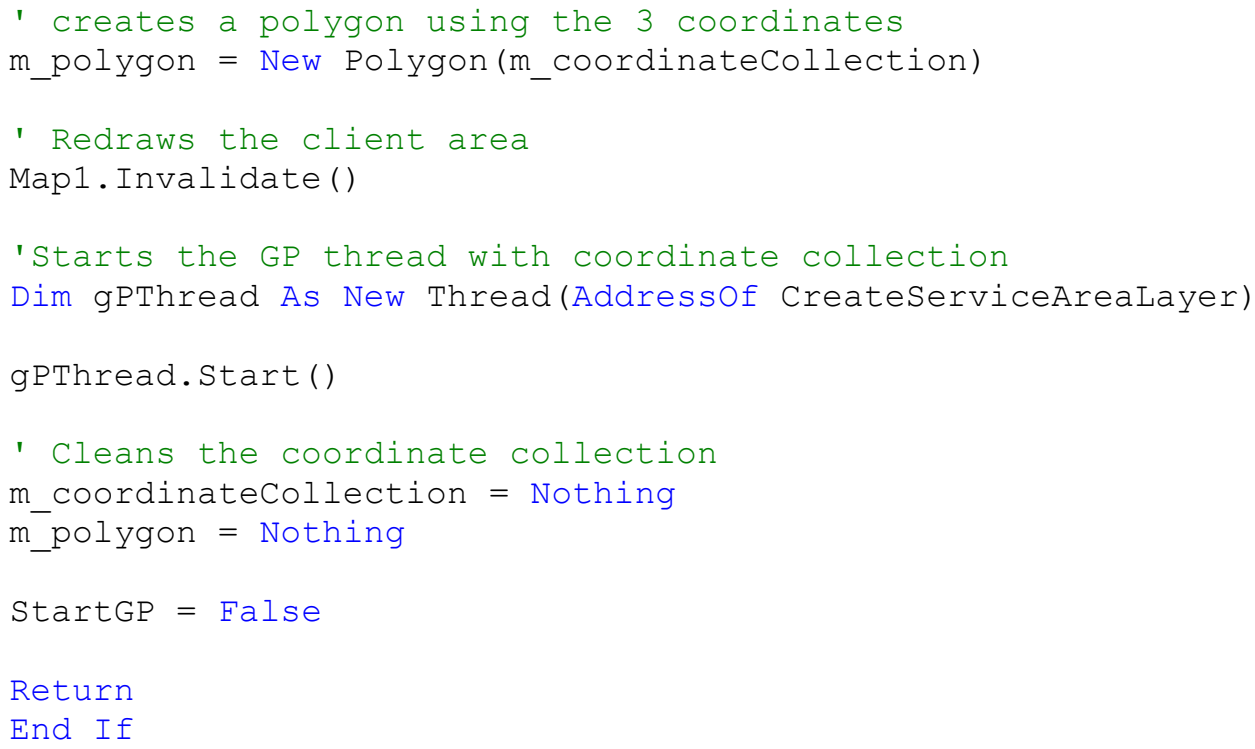

The coordinate collection is then passed to the custom web service, where it constructs a polygon array which serves as the input feature for the geoprocessing model on ESRI's sample server. The final output for this geoprocessing model is a string containing summary statistics based on the input polygon and the zonal statistics summary from the LandScan data set which can be seen in Figure 5.10.

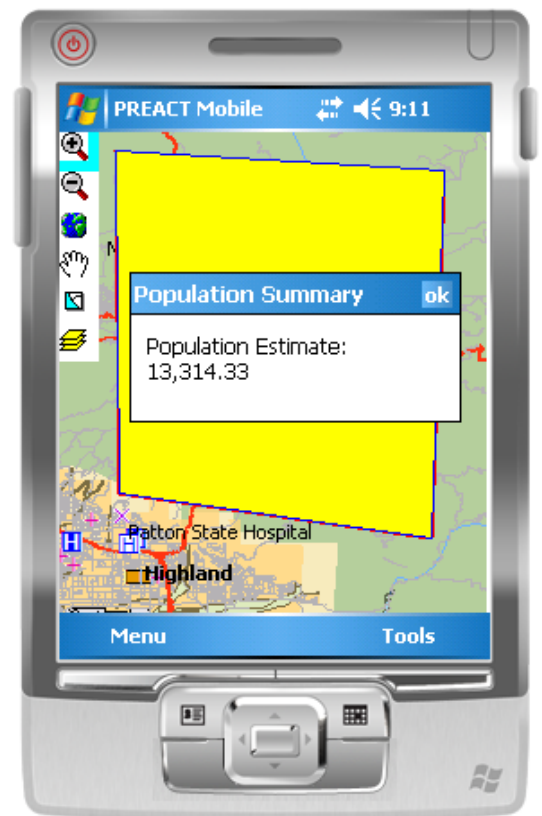

Figure 5.10 Final Project Output for Population Summary Analysis 


\section{Project Findings}

The development of analysis tools within the ArcGIS Server framework underlined the potential capabilities of such tools and their importance in Mobile GIS. Many known issues were found within the development framework of the Mobile SDK and ArcGIS Server that imposed numerous road blocks to the development of the analytical tools.

Although these issues hindered some of the project development, the tools have proven to be promising for future development of analytical capabilities within the mobile SDK for disaster responders.

\subsection{Incorporating Spatial Analysis in Mobile GIS}

Two paths can be taken within the ArcGIS server framework to implement spatially-based analytical tools for mobile users. This project focused on incorporating geoprocessingbased services for use with mobile clients, while geometry-based tools that could run in a disconnected environment were also examined. Both offer strong advancements in the current state of the typical mapping capabilities within the ArcGIS mobile SDK. From the research and development done within this project, the possibility of including these server-based geoprocessing tools offers insight into the ability to have tools that were once only available to desktop and web users deployed to the field. These geoprocessing services also allow the mobile user access to extensions within the desktop environment, such as Network Analyst, which was used in the pre-prototype implementation.

Although the advancement in mobile technology is evident, these tools are not ready to be implemented in disaster response. This prototype project it is not yet stable enough to deploy in situations where speed, functionality, and reliability are required. Significant testing of the mobile application and the web services required to incorporate the spatial analysis tools is needed to further assess how well this technology will perform in the field. This will require significant error handling for any interruptions in network connectivity as web services are being accessed. If any of the web services fail, the analytical tools will not function and troubleshooting can only be done on the server side, beyond control of the mobile users.

\subsubsection{Limitations of Geoprocessing Services in ArcGIS Server Mobile}

Since the functionality demonstrated is not fully tested, or out-of-the-box mobile analytical software available from ESRI, many limitations and issues were found. These limitations also apply to general development and deployment of ArcGIS Mobile applications (Table 6.1). 
Table 6.1 Limitations of ArcGIS Mobile Applications

\begin{tabular}{ll}
\hline Problem & Solution \\
\hline No support for ESRI geoprocessing services & $\begin{array}{l}\text { Create a custom web service which handles } \\
\text { communications }\end{array}$ \\
Services not always running on ArcGIS Server & $\begin{array}{l}\text { Need to implement checks for service } \\
\text { communication to limit runtime errors }\end{array}$ \\
$\begin{array}{l}\text { Limited cartographic abilities available on } \\
\text { mobile applications }\end{array}$ & $\begin{array}{l}\text { Eliminate any layers that are not supported and } \\
\text { design mobile caches with the cartographic } \\
\text { limitations in mind }\end{array}$ \\
\hline
\end{tabular}

\subsubsection{Triggering Geoprocessing Tasks from a Mobile Client}

Spatial analysis tools can be implemented within the ArcGIS Server Mobile SDK through the incorporation of a custom web service, which handles communication between a .NET Compact Framework-based mobile device and an ESRI geoprocessing service. This process allows for the task of triggering geoprocessing services residing on ArcGIS Server and then displaying the output on a mobile device. The implications of this are the ability to run complex spatial analytical tools, as well as to access data for inclusion in these tools that do not reside on the mobile client. This allows for the inclusion of robust tools accessed and run by disaster responders to better enable their collection processes.

\subsection{Incorporating Geoprocessing Services in PREACT}

The project results suggest that these tools are not yet suitable for a significant role in PREACT. Significant testing and tool development needs to be done before these analysis capabilities can be added to the mobile application within PREACT. Although testing was performed on the local server and mobile device, it is uncertain how these tools will operate once deployed. One concern is how long it will take for the tools to run once the mobile device and server are on two different networks. In project testing, the results from the server were sent back to the mobile device in under a minute. The new mobile application will need to be tested in an environment where the device and the server are not on the same server to acquire an accurate assessment of how quickly the tasks can be performed. Limited bandwidth situations (e.g. cellular networks) may also limit the capabilities of the tools.

\subsection{Future Work}

Time commitments and development issues did not allow for the entire project scope to be developed and tested for possible inclusion in the PREACT system. These tasks will be 
discussed as possible future work, based on their perceived value and level of effort to complete. The client will guide future work outside of this project but will closely reflect the findings of this prototype application.

The client has also expressed interest in bringing this technology to other applications within TEC. In particular, the Overseas Humanitarian Assistance Shared Information System (OHASIS) is a GIS Enterprise system which allows for the tracking and creation of humanitarian assistance projects through a web interface. The project incorporates mapping and a spatial database to keep track of the projects. Through this architecture, mobile users could use analytical tools while assessing areas for potential assistance projects and funding. Analyzing population parameters as well as other factors could aid in defining the potential issues within a given area seeking assistance.

\subsubsection{Data}

Although data requirements were driven by what was currently available in many Central American Countries, steps could be taken to showcase the technology if basic data collection processes occurred. In an attempt to drive data collection requirements, analytical tools could demonstrate the possibilities and added features of certain data types.

For example, standard addressing common in the United States would prove to be a costly and difficult task to replicate in Central America. A basic street network could be built where routing could occur between two input points. This would not produce the same results as an addressing street data set but it would allow for basic routing. This would greatly enhance the abilities of disaster responders to access areas and determine the best routes to key infrastructure and areas of concern.

\subsubsection{Analysis}

As a prototype system, only one key analytical tool was addressed in this project. In future work it will be important to incorporate existing analytical tools common to emergency response and to look into analytical tools that are focused on the field user.

\subsubsection{Building Local Analysis Tools for Use in a Disconnected Environment}

Although a primary focus of this project was to develop tools which did not rely on network connections, time constraints, as well as development issues, required this development to be postponed. These limitations do not overshadow the possibilities of developing these tools and their inclusion in future work will be considered. Several of these possible tools that will be worked on in future PREACT software builds are outlined below.

Potential Device-Based Analysis Tools:

- Select by Location 
The basis for this analysis will resemble the select by location tool in ArcMap and will use nearest neighbor functions to calculate distances to provide a list of selected features and their distances. The basis for this tool will be to allow the field user to find the nearest selected feature (i.e. hospitals, shelters, food stocks, etc). The inclusion of this tool will require multiple geoprocessing tasks that will run behind the scenes and will require both the use of the mobile cache and the ability to read each mobile layer from the cache for use in the geoprocessing tasks.

- Select by Attribute

The inclusion of this tool may be necessary for use with the select by location tools that allow for the selection of features in a single feature class. This tool may also prove useful for the user and may be incorporated into the system design.

- Buffer Tool

The ability to buffer features would allow field users to visualize distances for a quick proximity analysis of a given feature.

These tools would be implemented within the Mobile SDK through the geometry functions available. These geometry tools allow for basic spatially-based analysis, which would leverage the data residing in the map cache.

\subsubsection{Geoprocessing Analysis Tools}

Based on the work completed in this project, there are several considerations within the development of geoprocessing tasks for possible inclusion in mobile applications. These geoprocessing tasks are considered to be key developments in aiding field assessment teams to better do their jobs while working in the field.

Potential Mobile Geoprocessing Services:

- ALOHA - This third party software tool creates plume models for hazardous material. The inclusion of this tool will be the most difficult as it requires the use of the ALOHA software outside of the ArcGIS Server framework. Kevin Johnson has worked with this software in the past and can provide further insight into its possible inclusion. An ALOHA run must be performed on the server so that the output of that model can then be used by the custom programming to overlay the output plume onto the ArcGIS Server map control.

- Shelter Location Model - This model was developed by a PREACT team member and is already in use on PREACT's web client. The inclusion of this tool will be beneficial and easy to implement. This model selects features from a critical infrastructure dataset that satisfy criteria for potential shelters. It then further subsets this selection based on those points that are outside of risk areas (flood and landslide zones).

- Routing - The inclusion of a network routing tool running on the server would be an invaluable tool to the mobile responder, allowing each user to create routes from their current location to a given feature. This tool would be a proof of concept tool, as our partner nations do not currently have the necessary data to support the tool. The tool would also require the use of ArcGIS Network Analyst, which is not currently in the PREACT architecture. 


\subsubsection{Mobile Analysis in Other Domains}

Mobile analytical capabilities can also be applied to other domains or uses where spatial questions are often asked by mobile users. Future research could leverage the tools and processes identified in this paper and be applied to these domains.

\subsubsection{Search and Rescue}

Mobile GIS could also be applied to search and rescue techniques for use by response teams and include analytical capabilities for use by personnel currently searching for missing persons. Terrain analysis, coupled with the location of all responders in the field, could be used to direct search teams where to go next or to show areas which have not yet been searched. Models could be developed which aid in this process and the current GPS positions of all responders could be fed into the model through web services interfacing with the mobile application.

\subsubsection{Road Network Analysis}

Navigation and routing techniques have become very popular over the last few years with the introduction of mobile GPS units. Although these GPS navigation units allow for basic routing between two points, there is potential to expand on this development and have more sophisticated analytical capabilities. In the all too common travelling salesman problem, where an end user needs to find the best route possible between multiple stops, mobile network analysis could be applied. The application of a routing model, which leverages ArcGIS Network Analyst, would allow users to input their planned stops for the day and a geoprocessing service could be accessed which returns an optimal route. This could also integrate GPS tracking and be re-run as certain variables change, such as traffic, and their current location as they travel along the intended path. 


\section{Summary and Conclusions}

The initial project requirements and planned development were found to be overestimated based on the difficulty of implementing server-based functionality for mobile devices. The planned device-based analytical tools, which would be geometry-based and run locally on the device, were set aside to accommodate the intense customization required to implement geoprocessing services. Although these tools are still a priority for the client, it was found to be more beneficial to implement server-based analytical capabilities, based on the implications of having server run tools available to field-based devices.

Although not all initial approaches were examined, there were significant gains made in the ability to bring spatial analysis to the field for disaster responders. This project demonstrated the processes required to develop these tools that leverage server-side geoprocessing tools from ArcGIS Mobile within the ESRI software suite.

\subsection{Conclusions}

The inclusion of analytical capabilities in mobile GIS can aid emergency responders in making more informed decisions. These GIS capabilities available to desktop users are not currently available as out-of-the-box functionality deployable to mobile GIS applications. This technology is new to mobile development and can be difficult to implement without the right software and necessary programming capabilities. This project outlined a process by which this can be done, but it is not yet capable of being easily deployed to the field by the typical ArcGIS Desktop user. The implementation of these tools demonstrated a series of advancements in the ArcGIS Mobile framework, which include the following:

- Provide web service-based analytical capabilities to disaster responders

- Provide actionable intelligence regarding population estimates to field collection teams

- Incorporate geoprocessing services in ArcGIS Mobile with the use of a web service that handles communication protocols

- Ability to consume multiple geoprocessing services from multiple host servers

Although the technology is currently difficult to implement, deploying spatial analytical tools is possible and offers a vast amount of information to the field user. The key will be to develop models that best use the given data available for emergency responders, allowing them to derive meaningful information from it in a spatial context. This goes back to a fundamental development within GIS that has not been fully implemented in mobile GIS. As technology advances, these models will be more easily accessed and used by field responders, enabling them to collect critical information on the ground. 


\section{References}

Bolstadt, P. (2005). GIS Fundamentals: A First Text on Geographic Information Systems (2nd ed.). Minnesota: Eider Press

Command, U. S. S. (2007, May 8, 2007). Fuerzas Aliadas Humanitarias (FAHUM) 2007. Retrieved November 5, 2008, from http://www.southcom.mil/AppsSC/news.php?storyId=401

Cova, T. J. (1999). GIS in Emergency Management. In D. W. Rhind (Ed.), Geographical Information Systems: Principles, Techniques, Applications, and Managemen (pp. 845-858): John Wiley \& Sons, New York.

Cutter, S. L. (2003). GI Science, Disasters, and Emergency Management. Transactions in GIS, 7(4), 439-445.

Department of Defense, U. (2005). Department of Defense Directive: Military Support for Stability, Security, Transition, and Reconstruction (SSTR) Operations. Retrieved January 22, 2008. from http://www.dtic.mil/whs/directives/corres/pdf/300005p.pdf.

ESRI. (2008(a)). ArcGIS Services Directory. Retrieved October, 2008, from http://sampleserver1.arcgisonline.com/ArcGIS/rest/services/Demographics/ESRI_Pop ulation_World/GPServer/PopulationSummary

ESRI. (2008(b)). Design and create Mobile maps. Retrieved August, 2008, from http://webhelp.esri.com/arcgisdesktop/9.3/index.cfm?TopicName=Design_and_create _Mobile_maps

ESRI. (2008(c)). Developing Mobile Applications. ESRI Resource Center, from http://resources.esri.com/help/9.3/ArcGISmobile/adf/mobile_start.htm

ESRI. (2008(d)). Building effective data models for field data collection. ArcGIS Desktop 9.3 Help, from

http://webhelp.esri.com/arcgisdesktop/9.3/index.cfm?TopicName=Building_effective _data_models_for_field_data_collection

ESRI. (2008(f)). Deploying maps to mobile devices. Retrieved October 20, 2008, from http://webhelp.esri.com/arcgisdesktop/9.3/index.cfm?TopicName=Deploying_maps_t o_mobile_devices

ESRI. (2008(g)). An overview of Mobile GIS. Retrieved November 1, 2008, from http://webhelp.esri.com/arcgisdesktop/9.3/index.cfm?TopicName=An_overview_of Mobile_GIS

ESRI. (2008(h)). ArcPad - Mobile GIS Software for Field Mapping Applications. Retrieved November 4, 2008, from http://www.esri.com/software/arcgis/arcpad/index.html 
ESRI. (2008(i)). ArcGIS Mobile - Build and Deploy Mobile Applications Powered by ArcGIS Server. Retrieved November 4, 2008, from http://www.esri.com/software/arcgis/arcgismobile/index.html

Google. (2008). Google Mobile Demo. Retrieved September 16, 2008, from http://www.google.com/mobile/default/maps/demo.html

Hecker, E. J., Irwin, W., Cottrell, D., \& Bruzewicz, A. (2000). Strategies for Improving Response and Recovery in the Future. Natural Hazards Review, 1(3), 161-170.

Johnson, R. (May 2000). GIS Technology for Disasters and Emergency Management [Electronic Version]. An ESRI White Paper, 12. Retrieved 1/28/2008, from http://esri.com/library/whitepapers/pdfs/disastermgmt.pdf

Jong-Woo Kim, S.-S. P., Chang-Soo Kim, and Yugyung Lee. (2004). The Efficient WebBased Mobile GIS Service System through Reduction of Digital Map. Paper presented at the International Conference on Computational Science and Its Applications.

Laboratory, O. R. N. (2007). LandScan. from http://www.ornl.gov/sci/landscan/index.html

Raheja, N., Ojha, R., \& Mallik, S. R. Role of internet-based GIS in effective natural disaster management. Retrieved January 22, 2008, from http://www.gisdevelopment.net/technology/gis/techgi0030.htm

TDC Group, I. (2008). Freeance Mobile. Retrieved November 4, 2008, from http://www.freeance.com/mobile

Tom Rodden, N. D., Jonathan Trevor, Adrian Friday, and Kevin Palfreyman. (2000). Exploiting Space and Location as a Design Framework for Interactive Mobile Systems. ACM Transactions on Computer-Human Interaction, 7(3), 285-321.

Tsou, M.-H. (2004). Integrated Mobile GIS and Wireless Internet Map Servers for Environmental Monitoring and Managemant. Cartography and Geographic Information Science, 31(3), 153-165.

Vivioni, E. R., \& Camilli, R. (2003). Real-Time Streaming of Field Data. Computers and Geosciences, 29, 457-468.

Winnie S. M. Tang, J. R. S. (2003). Mobile Geographic Information Services (M-GIS): Technology That Changes the Way We Navigate Our World. Journal of Geospatial Engineering, 5(2), 43-50. 
Figures:

ESRI. (2008(a)). ArcGIS Services Directory. Retrieved October, 2008, from http://sampleserver1.arcgisonline.com/ArcGIS/rest/services/Demographics/ESRI_Pop ulation_World/GPServer/PopulationSummary

ESRI. (2008). "Developing Mobile Applications." ESRI Resource Center, from http://resources.esri.com/help/9.3/ArcGISmobile/SDK/mobile_start.htm. 


\section{Appendix A}

Mobile Recommendations FAHUM 08

\section{Mobile Recommendations:}

- Ability to edit field assessments

- $\quad$ Change the column width for the notes field in the selection page (if possible)

- $\quad$ Sketch capabilities

- Add time stamp to each assessment that is accessible through the 'select interface'

- $\quad$ Add multiple coordinate entries (MGRS, UTM, etc)

- Add more notes fields (possibly at the end of each tab)

- Need a dynamic screen capability to make use of the HP Smartphone's? Currently you have to scroll on the map screen, and the bottom of the assessment forms is cut off because there is no scroll bar.

- GET RID OF "OK" button on the top of the form and main display! Several times we had users click on the "ok" button resulting in the current window closing, which in the case of the assessment form automatically saves the assessment.

- Force a user login and write that information to each assessment

- Needed on the Assessment form:

○ Total population counts (while they may not be in need of immediate services a estimate of total people in the village/area would be very beneficial)

- Hazard Classification

- More detailed information could be obtained from the USAID Field Operations Guide 


\section{Appendix B}

Project Schedule

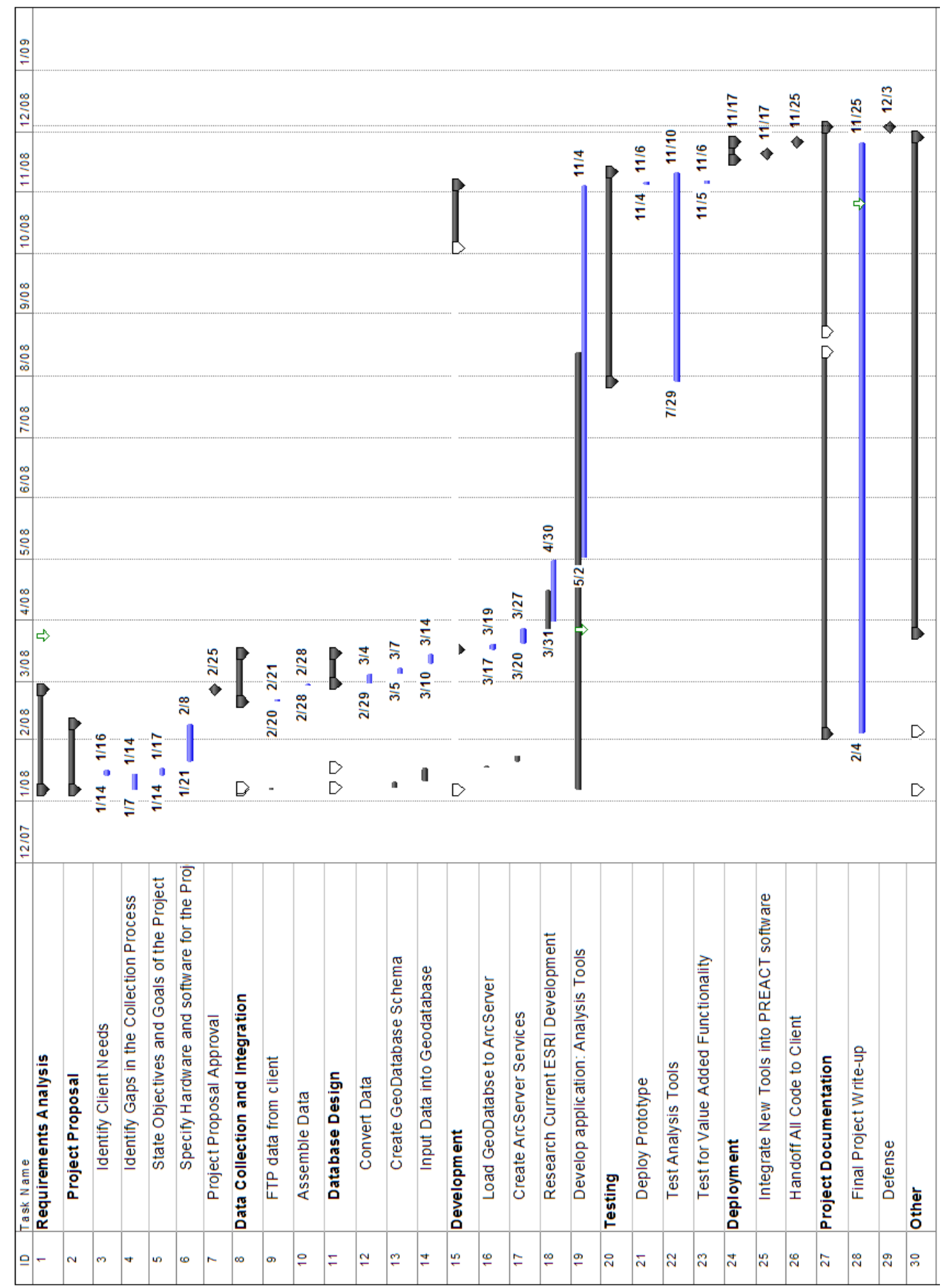




\section{Appendix C}

\section{ArcGIS Mobile Layer support (ESRI 2008)}

\section{ArcGIS Layer Type}

Feature Layer (Point, Line,

Polygon)

Raster Layers

Annotation Layer

Map Graphics Layer

Geoprocessing Tool Layer

IMS Feature Layer

Image Service Layers (ArcIMS,

ArcWebServices)

CAD Feature Layer

CAD Layer File, CAD

Annotation Layer

Raster Catalog Layer

Topology Layer

Archive Layer

Network Analysis Layers

Data Interoperability Layers

WMS Layer

WFS Layer

OGC Layer

Terrain Layer

Tin Layer

Coverage Annotation Layers

Dimension Layer

Tracking Layer

Representation Layer

Schematics Layer

\section{Supported}

Yes

Yes

Yes

No

Yes

Yes

No

Yes

No

No

No

Yes

No

Yes

No

Yes

Yes

No

No

No

No

No

No

No

\section{Notes}

Text only support. Scale dependency is not supported at the subclass level.

Graphic objects stored on the map display are not supported.

The output of the geoprocessing tool is supported.

Image service layers are not supported.

CAD entities represented as point, line and polygon feature layers are supported.

CAD text and the layer file itself is not supported.

Feature layers that participate in a topology are supported.

Network analysis layers are dynamic and results are stored in memory. They are not supported.

All direct read layers are supported.

Using the Data Interoperability Extension WMS is supported.

Using Data Interoperability Extension only

Using the Data Interoperability Extension only. Breaklines and Mass Points feature layers are supported.

Feature layers cannot use representation rendering functionality. Overrides are not supported. 


\section{Appendix D}

\section{Custom Web Service Code (C\#)}

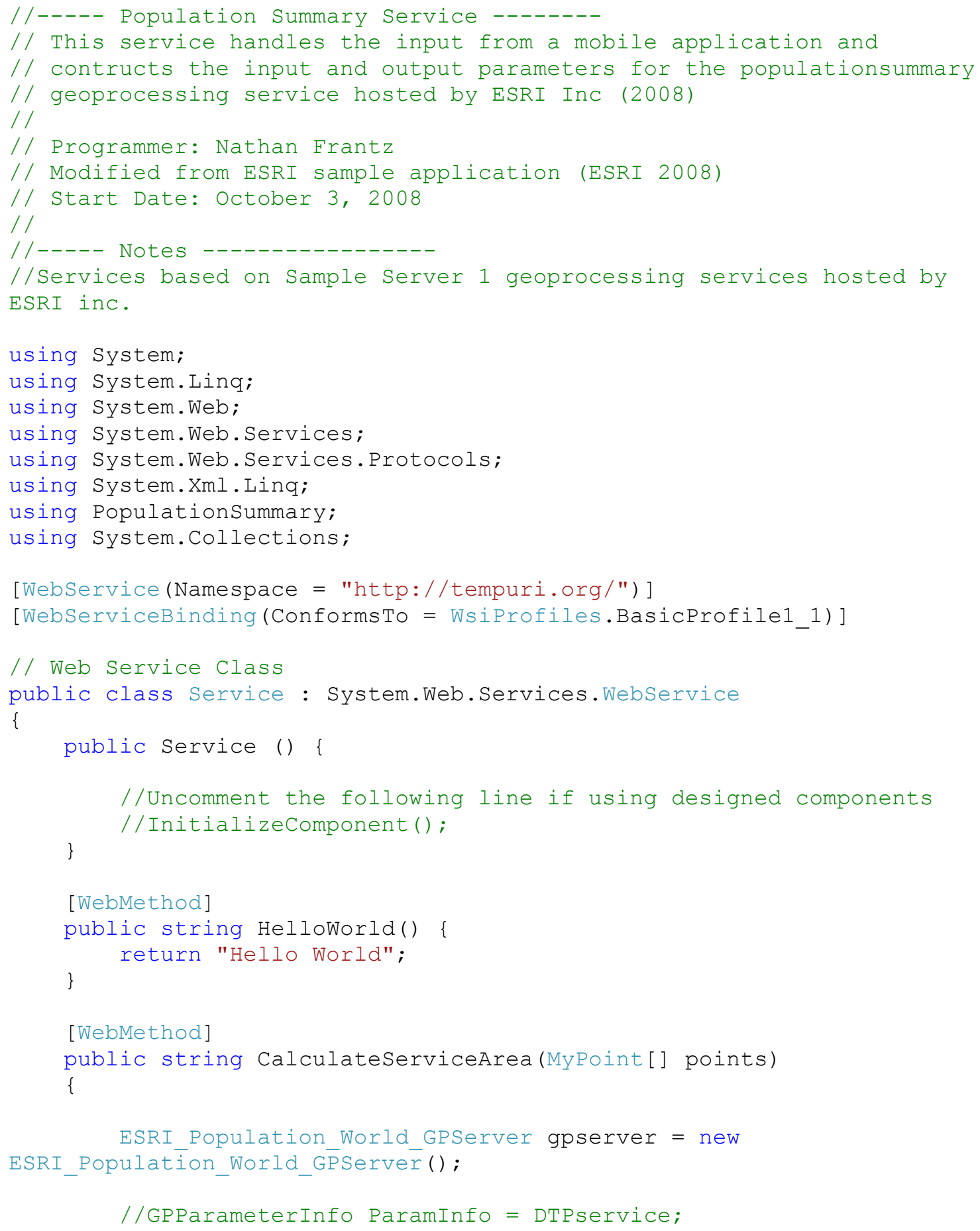




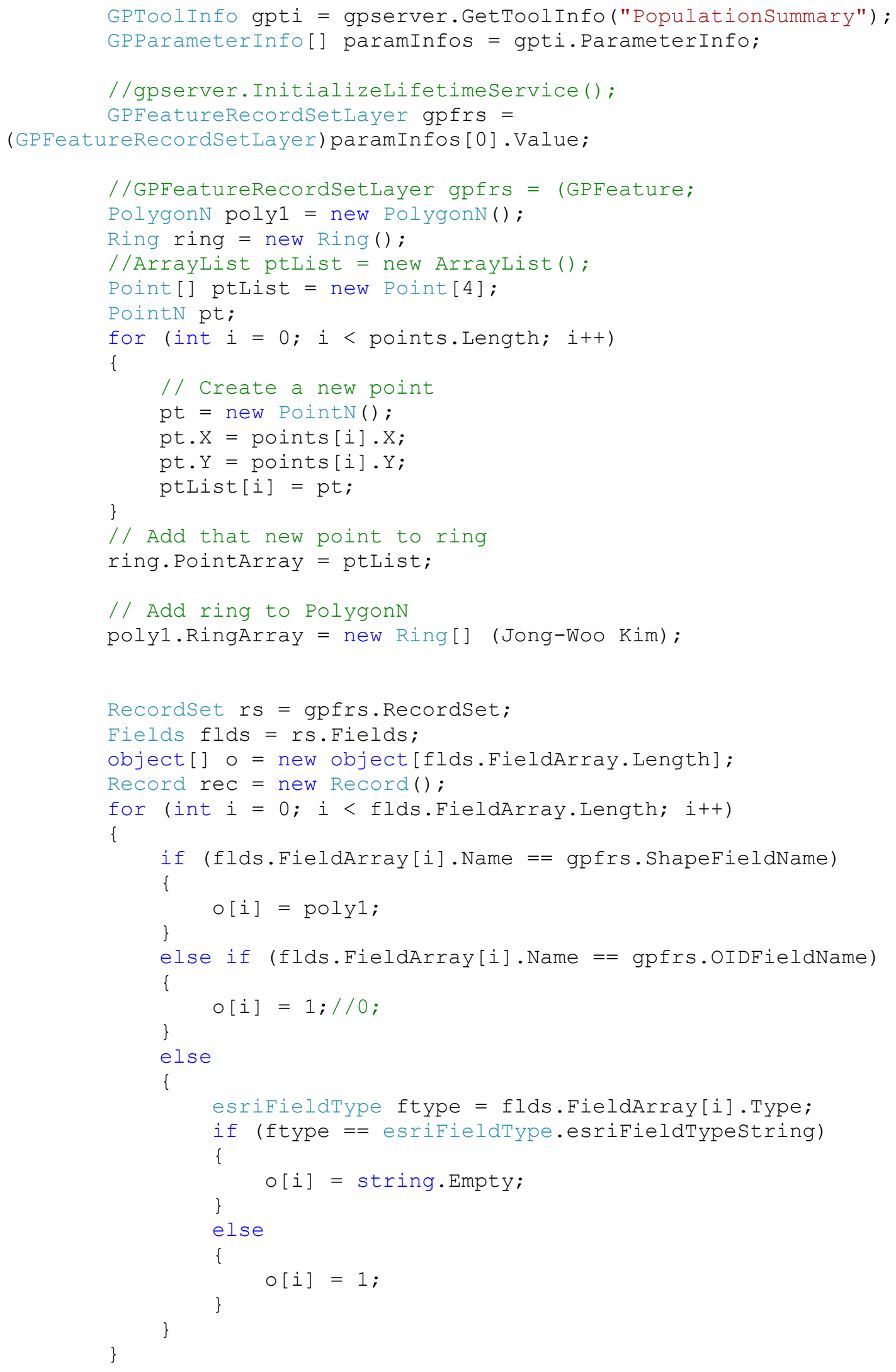




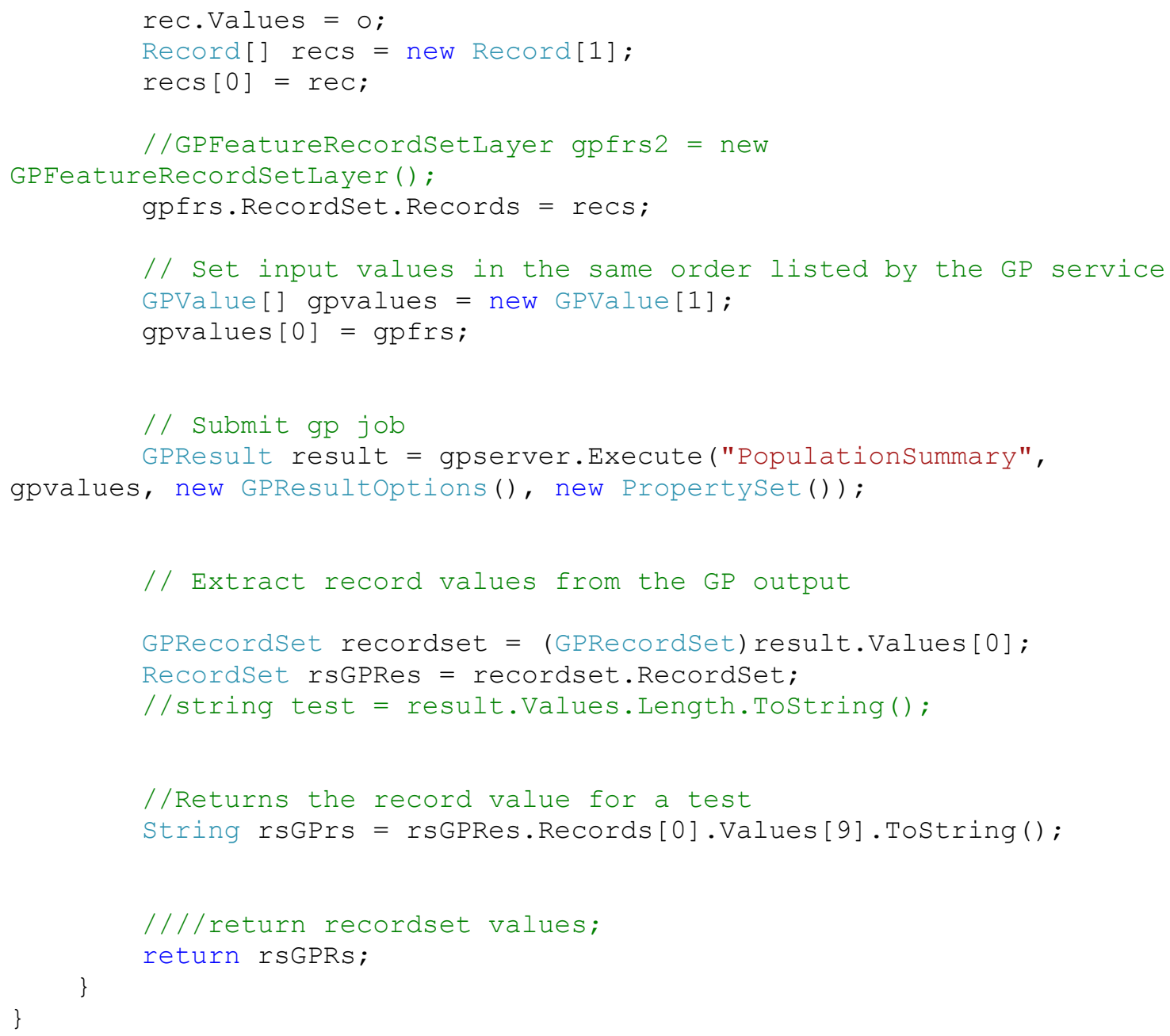




\section{Appendix E}

\section{Mobile Application Code (VB.NET)}

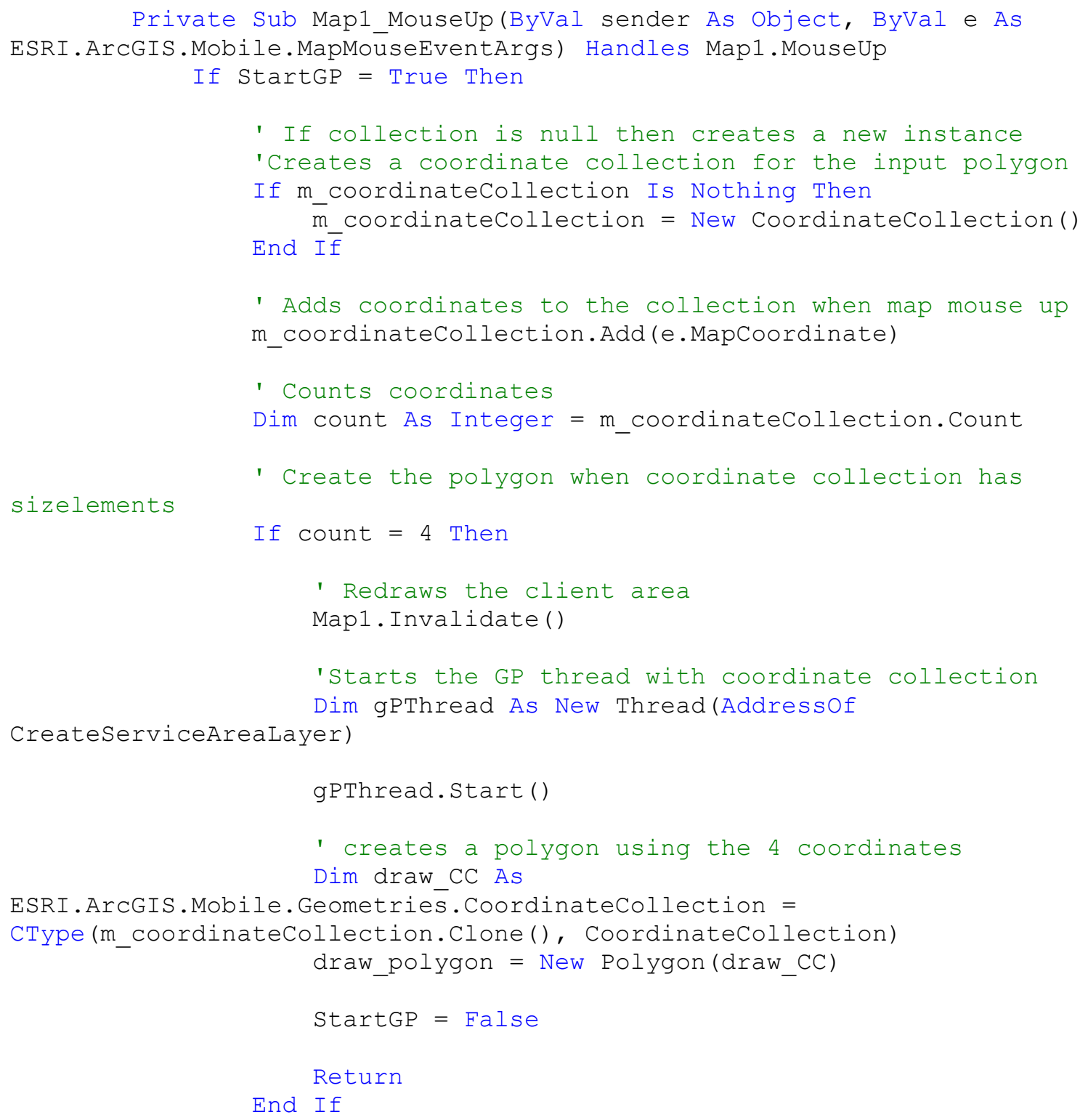


' Redraws the client area

Map1. Invalidate ()

End If

End Sub

Public Sub CreateServiceAreaLayer()

' Step 1: Put together an array of MyPoints

'Adds the points the array

Dim pts(3) As MyGPService.MyPoint

Dim ipt As Integer $=0$

For Each coord As ESRI.ArcGIS.Mobile.Geometries. Coordinate

In m_coordinatecollection

MyGPService.MyPoint ( )

Dim pt As MyGPService.MyPoint = New

pt. $X=\operatorname{coord} . X$

pt.Y $=$ coord.Y

pts $(i P t)=p t$

iPt $+=1$

Next

' Step 2: Invoke the custom web method

Dim proxy As New MyGPService.Service()

Dim sResult As String = proxy.CalculateServiceArea (pts)

' Step 3: Gather and handle the results

MessageBox.Show ("Population Estimation: " + sResult,

"Population Summary")

' Cleans the coordinate collection

m_coordinatecollection = Nothing

End Sub

Private Sub mnuPop_Click(ByVal sender As System.object, ByVal e As System.EventArgs) Handles mnuPop.click

'Sets a boolean operator which allowes for the analysis to

be triggered on a mouse up event

StartGP = True

'Clears the draw polygon variable

draw_polygon = Nothing

End Sub

Private Sub Mapl_Paint(ByVal sender As Object, ByVal e As

ESRI.ArcGIS.Mobile.MapPaintEventArgs) Handles Mapl.Paint

' Draws the coordinate collection to provide some feedback

If m_coordinatecollection IsNot Nothing Then 


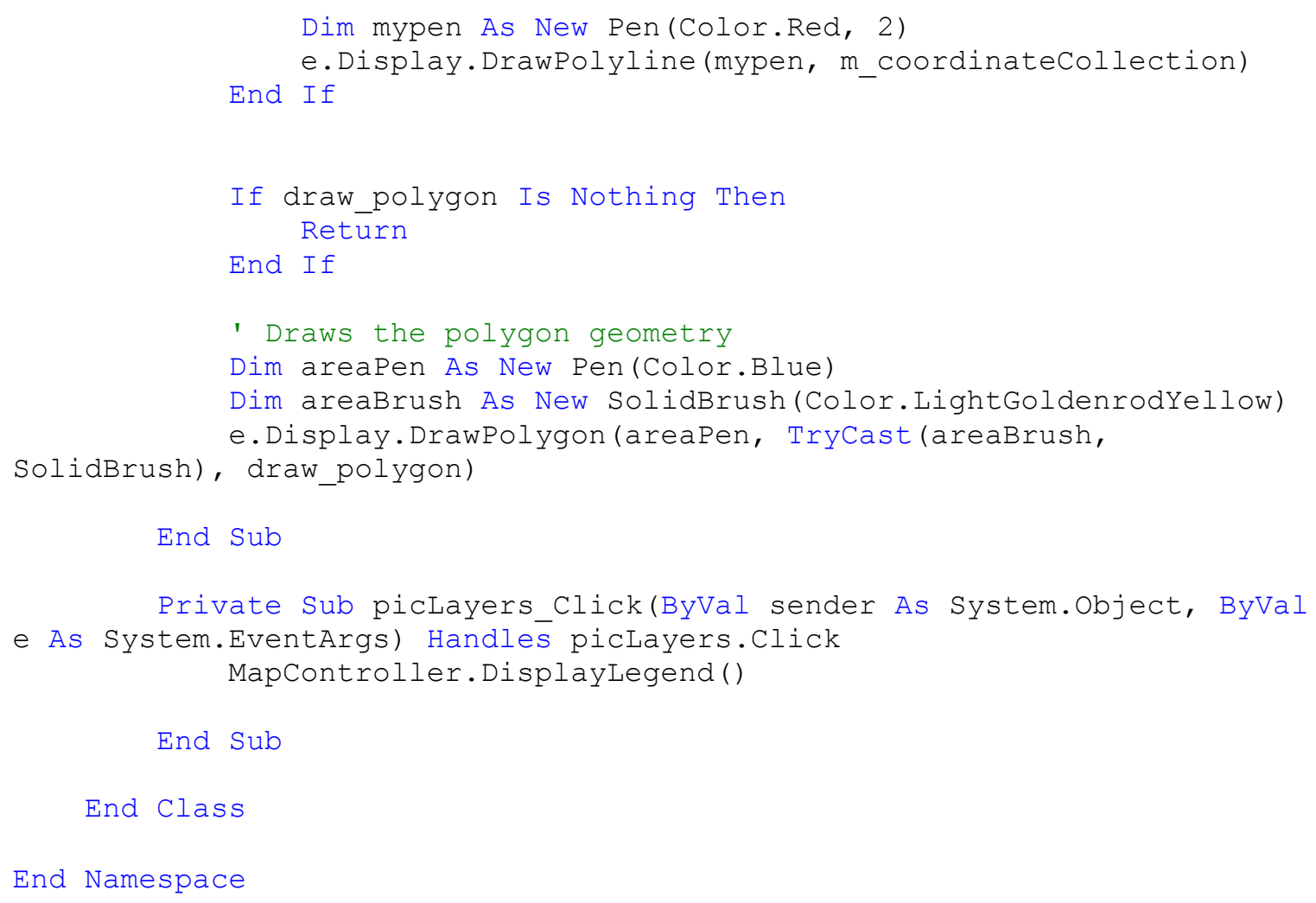

